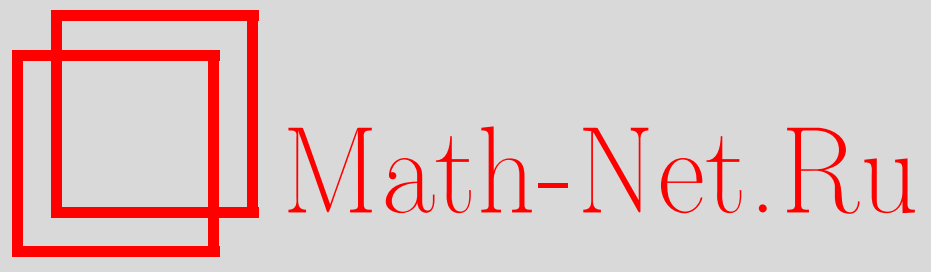

В. В. Горяйнов, Полугруппы аналитических функций в анализе и приложениях, $У М H, 2012$, том 67, выпуск $6,5-52$

DOI: https://doi.org/10.4213/rm9500

Использование Общероссийского математического портала Math-Net.Ru подразумевает, что вы прочитали и согласны с пользовательским соглашением http://www .mathnet.ru/rus/agreement

Параметры загрузки:

IP : 54.224 .187 .69

26 апреля 2023 г., 14:51:55

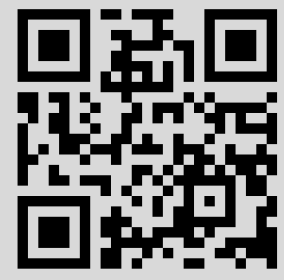




\section{Полугруппы аналитических функций в анализе и приложениях}

\section{В. В. Горяйнов}

Рассматриваются задачи анализа и некоторых смежных областей, в которых естественным образом возникают полугруппы аналитических функций относительно операции композиции. Основное внимание уделяется голоморфным отображениям круга (или полуплоскости) в себя. Выделяется роль неподвижных точек, как в описании структуры полугрупп, так и в приложениях. Отмечаются взаимосвязи проблемы дробного итерирования с некоторыми задачами теории случайных ветвящихся процессов, а также некоторыми вопросами некоммутативной вероятности. Показана роль инфинитезимального описания полугрупп конформных отображений в развитии параметрического метода теории однолистных функций.

Библиография: 94 названия.

Ключевые слова: однопараметрическая полугруппа, инфинитезимальная образующая, эволюционное семейство, эволюционное уравнение, дробные итерации, функция Кёнигса, неподвижные точки.

СОДЕРЖАНИЕ

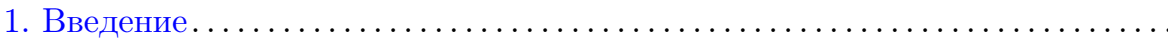

2. Однопараметрические полугруппы и инфинитезимальные преобразова-

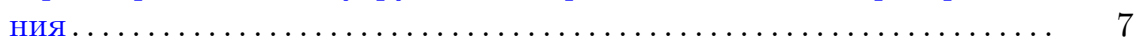

3. Задача вложения и функция Кёнигса......................... 12

4. Эволюционные семейства и эволюционное уравнение.............. 19

5. Параметрический метод теории однолистных функций ............ 25

6. Полугруппы вероятностных производящих функций и ветвящиеся про-

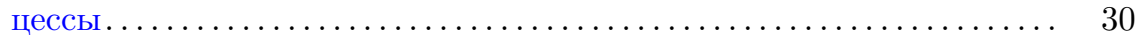

6.1. Задача вложения.................................... 30

6.2. Инфинитезимальные преобразования и безграничная делимость в полугруппе вероятностных производящих функций ....... 35

7. Инверсированное преобразование Коши

и некоммутативная вероятность ..................... 43

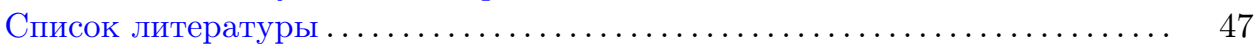

Работа выполнена при поддержке РФФИ (грант № 12-01-00434-а). 


\section{1. Введение}

Полугруппы аналитических функций относительно операции композиции естественно возникают в различных вопросах анализа и его приложениях. Так проблема дробного итерирования, исследование которой имеет богатую историю и восходит к работам Шрёдера [1] 1871 г. и Кёнигса [2] 1884 г., может рассматриваться как задача вложения натуральных итераций в однопараметрическую полугруппу. В теории случайных ветвящихся процессов итерации вероятностной производящей функции описывают динамику процесса Гальтона-Ватсона, т. е. марковского ветвящегося процесса с дискретным временем. Задача же вложения итераций в однопараметрическую полугруппу вероятностных производящих функций эквивалентна вложению процесса Гальтона-Ватсона в однородный марковский ветвящийся процесс с непрерывным временем. Задача вложения инверсированного преобразования Коши вероятностного распределения в однопараметрическую полугруппу возникает в некоммутативной теории вероятностей в связи с аналогами формулы Леви-Хинчина для безгранично делимых законов. Задача вложения итераций голоморфного отображения единичного круга в себя в однопараметрическую полугруппу возникает также в теории композиционных операторов.

Другое направление исследований, связанное с полугруппами аналитических функций, имеет своим источником классическую работу Лёвнера [3] 1923 г. В этой работе Лёвнер заложил основы параметрического метода геометрической теории функций и впервые получил продвижение в решении проблемы коэффициентов, которую в качестве гипотезы высказал Бибербах [4] в 1916 г. Эта проблема на десятилетия определила направление развития теории однолистных функций и была решена де Бранжем [5], [6] в 1984 г. с использованием параметрического метода Лёвнера. Подход, который систематически развивал Лёвнер (см. [7]-[10]), основывался на представлении элемента полугруппы посредством композиции инфинитезимальных преобразований, что в идейном плане роднит его с теорией групп Ли.

В настоящей работе устанавливается полный аналог формулы БерксонаПорты для инфинитезимальной образующей однопараметрической полугруппы голоморфных отображений единичного круга в себя с заданными неподвижными точками. Решается задача вложения голоморфного отображения в однопараметрическую полугруппу в терминах функции Кёнигса. Отмечается связь задачи вложения и проблемы дробных итераций с различными вопросами теории конформного отображения, теории ветвящихся процессов, некоммутативной вероятности. Устанавливается эквивалентность условий вложимости и безграничной делимости в полугруппе вероятностных производящих функций.

Автор выражает искреннюю признательность Е. М. Чирке и участникам семинара по комплексному анализу МИ РАН за неоднократные плодотворные обсуждения результатов, Б. А. Севастьянову, А. М. Зубкову и В. А. Ватутину за обсуждение задач теории ветвящихся процессов. Неоценимую помощь при подготовке рукописи оказал С.П. Суетин, которому автор также выражает свою благодарность. 


\section{2. Однопараметрические полугруппы и инфинитезимальные преобразования}

Проблема дробного итерирования для заданной функции $f(z)$ заключается в отыскании семейства функций $f^{t}(z), t \geqslant 0$, удовлетворяющего условиям: $f^{1}(z)=f(z), f^{0}(z)=z$ и $f^{t+s}(z)=f^{t} \circ f^{s}(z)$ для всех $s, t \geqslant 0$. Эта задача имеет богатую историю и изучалась в различных постановках многими авторами. Первые исследования касались локального случая, когда сама функция $f$ и ее итерации определялись степенными рядами лишь в окрестности неподвижной точки $a, f(a)=a$. Еще Кёнигс [2] показал, что если $\left|f^{\prime}(a)\right|$ не равняется ни 0 , ни 1 , то $f$ можно вложить в семейство дробных итераций. Позже получило развитие направление, связанное с изучением целых и мероморфных функций. Здесь результаты оказались диаметрально противоположными локальному случаю. Так в работах Бейкера [11], а также Карлина и Макгрегора [12] было показано, что вложимость в этом случае при достаточно общих предположениях возможна лишь для дробно-линейных функций. Отметим также работы [13], [14] и монографии [15], [16], которые содержат обзоры работ по данной тематике и отражают связь задачи дробного итерирования с функциональными уравнениями. Кроме того, как заметил Адамар [17], существенное продвижение в исследовании проблемы дробного итерирования было получено, когда стали рассматривать семейство дробных итераций как однопараметрическую полугруппу и описывать ее посредством инфинитезимальной образующей.

Связь проблемы дробного итерирования с задачей вложения процесса Гальтона-Ватсона в однородный марковский ветвящийся процесс с непрерывным временем, а также некоторые задачи теории композиционных операторов и теории конформного отображения стимулировали изучения случая, когда сама функция и ее итерации отображают единичный круг (или верхнюю полуплоскость) в себя. При этом часто требуется, чтобы все итерации наследовали некоторые свойства самой функции.

Пусть $\mathfrak{P}$ - совокупность всех голоморфных в единичном круге $\mathbb{D}=\{z \in \mathbb{C}$ : $|z|<1\}$ функций $f$, принимающих значения из $\mathbb{D}$, т. е. $f(\mathbb{D}) \subseteq \mathbb{D}$. Очевидно, $\mathfrak{P}$ образует топологическую полугруппу относительно операции композиции и топологии локально равномерной сходимости в $\mathbb{D}$. Роль единицы в этой полугруппе играет тождественное преобразование $f(z) \equiv z$. Кроме того, $\mathfrak{P}$ является выпуклым множеством в линейном топологическом пространстве $\mathscr{H}(\mathbb{D})$ голоморфных в $\mathbb{D}$ функций с топологией локально равномерной сходимости.

Рассматривая $\mathbb{R}^{+}=\{t \in \mathbb{R}: t \geqslant 0\}$ как аддитивную полугруппу с обычной топологией вещественных чисел, под однопараметрической полугруппой в $\mathfrak{P}$ будем понимать непрерывный гомоморфизм $t \mapsto f^{t}$, действующий из $\mathbb{R}^{+}$в $\mathfrak{P}$. Это означает, что семейство $\left\{f^{t}\right\}_{t \geqslant 0}$ функций из $\mathfrak{P}$ удовлетворяет условиям:

(i) $f^{t+s}(z)=f^{t} \circ f^{s}(z)$ при $s, t \geqslant 0$;

(ii) $f^{t}(z) \rightarrow z$ локально равномерно в $\mathbb{D}$ при $t \rightarrow 0$.

Заметим, что при целых неотрицательных значениях $t$ мы получаем обычные итерации $f^{n}=f \circ f^{n-1}, n=2,3, \ldots$, функции $f=f^{1}$. Поэтому элементы семейства $\left\{f^{t}\right\}_{t \geqslant 0}$ называют также дробными итерациями функции $f$. Таким образом, проблема дробных итераций для $f \in \mathfrak{P}$ состоит в вопросе существования такой однопараметрической полугруппы $t \mapsto f^{t}$ в $\mathfrak{P}$, для которой $f^{1}=f$. 
Однопараметрические полугруппы играют важную роль в изучении структуры самой полугруппы $\mathfrak{P}$ и ее подполугрупп.

Как правило, синтез алгебраических и топологических свойств приводит к достаточно жестким конструкциям. Иллюстрацией этого может служить бесконечная дифференцируемость по $t$ семейства функций $f^{t}(z)$ однопараметрической полугруппы $t \mapsto f^{t}$ в $\mathfrak{P}$. Действительно, зафиксируем произвольно $r \in(0,1)$ и покажем, что для любого $z \in K_{r}=\{\zeta \in \mathbb{C}:|z| \leqslant r\}$ функция $t \mapsto f^{t}(z)$ является бесконечно дифференцируемой в некоторой окрестности точки $t=0$. Условие (i) распространит тогда свойство дифференцируемости на всю полуось $(0, \infty)$.

Пусть $\eta$ - бесконечно дифференцируемая на $\mathbb{R}$ функция, обращающаяся в нуль вне интервала $(0,1)$ и удовлетворяющая условиям: $\eta(t)>0$ при $t \in(0,1)$ и

$$
\int_{-\infty}^{\infty} \eta(t) d t=\int_{0}^{1} \eta(t) d t=1
$$

Для $\delta>0$ через $\eta_{\delta}$ будем обозначать функцию $\eta_{\delta}(t)=\eta(t / \delta)$. Распорядимся теперь выбором $\delta>0$ следующим образом. Фиксируем $\rho \in(r, 1)$ и выберем $\delta>0$ так, чтобы при $t \in(0, \delta)$ выполнялись условия:

(a) $\left|f^{t}(z)\right| \leqslant \rho$ при $z \in K_{r}$;

(b) $\operatorname{Re}\left\{\left(f^{t}\right)^{\prime}(z)\right\}>1 / 2$ при $z \in K_{\rho}$.

Это можно сделать, поскольку $f^{t}(z) \rightarrow z$ и $\left(f^{t}\right)^{\prime}(z) \rightarrow 1$ локально равномерно в $\mathbb{D}$ при $t \rightarrow 0$. Определим теперь функцию

$$
F(z)=\int_{-\infty}^{\infty} \eta_{\delta}(t) f^{t}(z) d t=\int_{0}^{\delta} \eta_{\delta}(t) f^{t}(z) d t .
$$

Очевидно, что $F$ является голоморфной в $\mathbb{D}$ функцией. Кроме того, при $z \in K_{\rho}$ выполняется условие

$$
\operatorname{Re} F^{\prime}(z)=\int_{0}^{\delta} \eta_{\delta}(t) \operatorname{Re}\left\{\left(f^{t}\right)^{\prime}(z)\right\} d t \geqslant \frac{1}{2} \int_{0}^{\delta} \eta_{\delta}(t) d t=\frac{\delta}{2}>0,
$$

из которого следует однолистность $F$ в круге $K_{\rho}$. Введем также в рассмотрение функцию

$$
\Phi(z, t)=F\left(f^{t}(z)\right)
$$

$z \in \mathbb{D}, t \geqslant 0$. Очевидные преобразования

$$
\Phi(z, t)=\int_{-\infty}^{\infty} \eta_{\delta}(s) f^{s}\left(f^{t}(z)\right) d s=\int_{-\infty}^{\infty} \eta_{\delta}(s) f^{s+t}(z) d s=\int_{-\infty}^{\infty} \eta_{\delta}(\tau-t) f^{\tau}(z) d \tau
$$

показывают, что $\Phi(z, t)$ является бесконечно дифференцируемой по $t$.

Далее, в силу условия (а) точка $f^{t}(z)$ попадает в круг $K_{\rho}$ при $z \in K_{r}$ и $t \in[0, \delta]$. Поэтому для этих $t$ и $z$ можно записать

$$
f^{t}(z)=F^{-1}(\Phi(z, t))
$$

откуда следует бесконечная дифференцируемость функции $t \mapsto f^{t}(z)$ в интервале $(0, \delta)$ для любого $z \in K_{r}$. При этом

$$
\begin{aligned}
\frac{\partial}{\partial t} f^{t}(z) & =-\frac{1}{F^{\prime}\left(f^{t}(z)\right)} \int_{-\infty}^{\infty} \eta_{\delta}^{\prime}(\tau-t) f^{\tau}(z) d \tau \\
& =-\frac{1}{F^{\prime}\left(f^{t}(z)\right)} \int_{-\infty}^{\infty} \eta_{\delta}^{\prime}(s) f^{s}\left(f^{t}(z)\right) d s=v\left(f^{t}(z)\right)
\end{aligned}
$$


где

$$
v(z)=-\frac{1}{F^{\prime}(z)} \int_{-\infty}^{\infty} \eta_{\delta}^{\prime} f^{\tau}(z) d \tau=\left.\frac{\partial}{\partial t} f^{t}(z)\right|_{t=0} .
$$

Поскольку $r$ выбиралось произвольным в интервале $(0,1)$, то функция

$$
v(z)=\left.\frac{\partial}{\partial t} f^{t}(z)\right|_{t=0}=\lim _{t \rightarrow 0} \frac{f^{t}(z)-z}{t}
$$

определена и голоморфна во всем единичном круге $\mathbb{D}$. Эту функцию будем называть инфинитезимальной образующей однопараметрической полугруппы $t \mapsto f^{t}$ или инфинитезимальным преобразованием полугруппы $\mathfrak{P}$. Инфинитезимальная образующая вполне характеризует однопараметрическую полугруппу $t \mapsto f^{t}$ посредством дифференциального уравнения

$$
\frac{\partial}{\partial t} f^{t}(z)=v\left(f^{t}(z)\right)
$$

и начального условия $\left.f^{t}(z)\right|_{t=0}=z$.

Впервые вид инфинитезимальной образующей $v$ однопараметрической полугруппы $t \mapsto f^{t}$ в $\mathfrak{P}$ дал Лёвнер [3] в случае, когда все функции $f^{t}$ однопараметрической полугруппы оставляют неподвижной точку $z=0$, т. е. $f^{t}(0)=0, t \geqslant 0$. В этом случае $v(z)=-z p(z)$, где $p$ - голоморфная в $\mathbb{D}$ функция с неотрицательной вещественной частью. Этот результат легко преобразовать на случай, когда функции $f^{t}$ однопараметрической полугруппы $t \mapsto f^{t}$ оставляют неподвижной произвольную точку $a \in \mathbb{D}$. Действительно, в этом случае функции $g^{t}=L \circ f^{t} \circ L^{-1}$, где

$$
L(z)=\frac{z-a}{1-\bar{a} z}
$$

также образуют однопараметрическую полугруппу $t \mapsto g^{t}$ в $\mathfrak{P}$ и $g^{t}(0)=0, t \geqslant 0$. При этом, если

$$
\left.\frac{\partial}{\partial t} g^{t}(z)\right|_{t=0}=-z p(z)
$$

Tо

$$
\left.\frac{\partial}{\partial t} f^{t}(z)\right|_{t=0}=\left.\frac{1}{L^{\prime}(z)} \frac{\partial}{\partial t} g^{t}(L(z))\right|_{t=0}=-\frac{L(z)}{L^{\prime}(z)} p(L(z))=(a-z)(1-\bar{a} z) \frac{p(L(z))}{1-|a|^{2}} .
$$

Другими словами, если однопараметрическая полугруппа $t \mapsto f^{t}$ в $\mathfrak{P}$ оставляет неподвижной точку $a \in \mathbb{D}$, то ее инфинитезимальная образующая имеет вид

$$
v(z)=(a-z)(1-\bar{a} z) p(z),
$$

где $p$ - голоморфная в $\mathbb{D}$ функция с неотрицательной вещественной частью.

Заметим, что если $f \in \mathfrak{P}$ и $f(z) \not \equiv z$, то в силу принципа гиперболической метрики (см., например, [18], [19]) в $\mathbb{D}$ может быть не более одной неподвижной точки. С другой стороны, функция $f \in \mathfrak{P}$ может вовсе не иметь неподвижных точек внутри единичного круга $\mathbb{D}$. В связи с этим важную роль играет результат, установленный Данжуа [20] и Вольфом [21]. 
Tеорема 1. Пусть $f \in \mathfrak{P}$ отлична от дробно-линейного преобразования единичного круга $\mathbb{D}$ на себя. Тогда существует единственная точка $q,|q| \leqslant 1$, такая, что последовательность натуральных итераций $f^{n}, n=1,2, \ldots$, сходится локально равномерно в $\mathbb{D} \kappa q$. При этом, если $|q|=1$, то существуют угловые предель

$$
\lim _{z \rightarrow q} f(z)=f(q), \quad \lim _{z \rightarrow q} f^{\prime}(z)=f^{\prime}(q)
$$

$u f(q)=q, 0<f^{\prime}(q) \leqslant 1$.

В литературе $q$ называют точкой Данжуа-Вольфа функции $f$. Если $q \in \mathbb{D}$, то очевидно, что $f(q)=q$ и $q$ является единственной неподвижной точкой в $\mathbb{D}$, а в силу обобщенной леммы Шварца $\left|f^{\prime}(q)\right| \leqslant 1$. Таким образом, точка Данжуа-Вольфа функции $f$ из $\mathfrak{P}$ является неподвижной притягивающей точкой. Кроме того, все итерации функции $f$ (включая дробные итерации, если они существуют) имеют одну и ту же точку Данжуа-Вольфа. В терминах этого понятия Берксон и Порта [22] дали полное описание инфинитезимальных преобразований полугруппы $\mathfrak{P}$.

Теорема 2. Для того чтобы голоморфная в $\mathbb{D}$ функиия $v$ представляла собой инфинитезимальную образующую однопараметрической полугруппь $t \mapsto f^{t}$ в $\mathfrak{P}$ с точкой Данжуа-Вольфа $q,|q| \leqslant 1$, необходимо и достаточно, чтобы она допускала представление в виде

$$
v(z)=(q-z)(1-\bar{q} z) p(z),
$$

где $p$ - голоморфная в $\mathbb{D}$ функиия с неотрищательной вещественной частью.

Равенство (1) известно в литературе как формула Берксона-Порты. Вопрос о том, как трансформируется формула (1) при наличии других неподвижных точек, исследовался многими авторами в различных постановках (см., например, [23]-[25]). Этот вопрос возникает также в теории композиционных операторов [26], [27].

Допустим, что $f \in \mathfrak{P}$ с точкой Данжуа-Вольфа $q,|q| \leqslant 1$, и оставляет также неподвижными точки $a_{1}, \ldots, a_{n}$. Как уже отмечалось, точки $a_{1}, \ldots, a_{n}$ должны быть расположены на единичной окружности $\mathbb{T}=\{z \in \mathbb{C}:|z|=1\}$, а условие того, что они являются неподвижными точками, понимается в смысле угловых пределов

$k=1, \ldots, n$.

$$
\lim _{z \rightarrow a_{k}} f(z)=a_{k}
$$

Теорема 3. Для того чтобы голоморфная в $\mathbb{D}$ функция $v$ представляла собой инфинитезимальную образующую однопараметрической полугруппь $t \mapsto f^{t}$ в $\mathfrak{P}$ с точкой Данжуа-Волъфа $q,|q| \leqslant 1$, и неподвижными точками $a_{1}, \ldots, a_{n} \in \mathbb{T}$, в которых функиии $f^{t}, t>0$, имеют конечные угловые производнье, необходимо и достаточно, чтобъ она допускала представление в виде

$$
v(z)=\frac{(q-z)(1-\bar{q} z)}{\sum_{k=1}^{n} \lambda_{k} \frac{1+\bar{a}_{k} z}{1-\bar{a}_{k} z}+p(z)},
$$

где $\lambda_{k}>0, k=1, \ldots, n$, а $p$ - голоморфная в $\mathbb{D}$ функиия с неотрицательной вещественной частью. 
ДокАзАтельство. Заметим вначале, что в силу результатов работы [24] (см. теорему 1 и лемму 2) голоморфная в $\mathbb{D}$ функция $v$ является инфинитезимальной образующей однопараметрической полугруппы $t \mapsto f^{t}$ в $\mathfrak{P}$ с точкой Данжуа-Вольфа $q,|q| \leqslant 1$, и неподвижной точкой $a \in \mathbb{T}$, в которой функции $f^{t}$, $t>0$, имеют конечные угловые производные, в том и только том случае, если она допускает представление в виде

$$
v(z)=\frac{(q-z)(1-\bar{q} z)}{\lambda \frac{1+\bar{a} z}{1-\bar{a} z}+g(z)},
$$

где $\lambda>0$, а функция $g$ голоморфна в $\mathbb{D}$ и имеет неотрицательную вещественную часть.

Допустим теперь, что $t \mapsto f^{t}$ - однопараметрическая полугруппа в $\mathfrak{P}$ с точкой Данжуа-Вольфа $q$ и неподвижными точками $a_{1}, \ldots, a_{n} \in \mathbb{T}$, в которых функции $f^{t}$ имеют конечные угловые производные, и пусть $v$ - инфинитезимальная образующая этой однопараметрической полугруппы. В силу сделанного выше замечания для каждого $k=1, \ldots, n$ найдутся $\alpha_{k}>0$ и функции $g_{k}$, голоморфные в $\mathbb{D}$ с неотрицательной вещественной частью, такие, что выполняются равенства

$$
v(z)=\frac{(q-z)(1-\bar{q} z)}{\alpha_{k} \frac{1+\bar{a}_{k} z}{1-\bar{a}_{k} z}+g_{k}(z)},
$$

$k=1, \ldots, n$. С другой стороны, инфинитезимальная образующая $v$ допускает представление (1), которое можно записать в виде

$$
v(z)=(q-z)(1-\bar{q} z) \frac{1}{g(z)},
$$

где $g$ - также голоморфная в $\mathbb{D}$ функция с неотрицательной вещественной частью. Но тогда для этой функции $g$ будут выполняться равенства

$$
g(z)=\alpha_{k} \frac{1+\bar{a}_{k} z}{1-\bar{a}_{k} z}+g_{k}(z)
$$

$k=1, \ldots, n$. Суммируя эти равенства по $k$ от 1 до $n$, получаем

$$
g(z)=\frac{1}{n} \sum_{k=1}^{n}\left(\alpha_{k} \frac{1+\bar{a}_{k} z}{1-\bar{a}_{k} z}+g_{k}(z)\right) .
$$

Таким образом, $v$ можно представить формулой (2), положив

$$
\lambda_{k}=\frac{\alpha_{k}}{n}, \quad p(z)=\frac{1}{n} \sum_{k=1}^{n} g_{k}(z) .
$$

Обратно, допустим, что $v$ имеет вид (2). Нам нужно показать, что $v$ является инфинитезимальной образующей однопараметрической полугруппы $t \mapsto f^{t}$ в $\mathfrak{P}$ с точкой Данжуа-Вольфа $q$, и неподвижными точками $a_{1}, \ldots, a_{n} \in \mathbb{T}$, в которых функции $f^{t}$ имеют конечные угловые производные. Это следует из замечания, сделанного выше, и того, что для каждого $k=1, \ldots, n$ функцию $v$ можно представить в виде (3), положив

$$
\alpha_{k}=\lambda_{k}, \quad g_{k}(z)=g(z)+\sum_{j \neq k} \alpha_{j} \frac{1+\bar{a}_{j} z}{1-\bar{a}_{j} z} .
$$

Теорема доказана. 


\section{3. Задача вложения и функция Кёнигса}

Как уже отмечалось выше, проблема дробных итераций для функции $f$ из $\mathfrak{P}$ состоит в возможности вложить ее в однопараметрическую полугруппу в $\mathfrak{P}$. Уже в первых исследованиях проблемы итераций (см., например, [17]) была обнаружена связь этой задачи с функциональными уравнениями Абеля и Шрёдера. Эта тема детально освещена с обширной библиографией в монографиях [15], [16] не только для аналитических функций.

Пусть $f \in \mathfrak{P}$ и $\Phi$ является решением уравнения Абеля

$$
\Phi(f(z))=\Phi(z)+1 .
$$

Тогда для всех натуральных итераций $f^{n}, n=1, \ldots, n$, функции $f$ будут выполняться равенства

$$
\Phi\left(f^{n}(z)\right)=\Phi(z)+n .
$$

Аналогичная ситуация наблюдается и для уравнения Шрёдера

$$
\Psi(f(z))=\gamma \Psi(z)
$$

где $\gamma$ - некоторая константа. Его решение $\Psi$ и итерации $f^{n}, n=1,2, \ldots$, будут удовлетворять соотношениям

$$
\Psi\left(f^{n}(z)\right)=\gamma^{n} \Psi(z) .
$$

В связи с этим изучались способы решения функциональных уравнений Абеля и Шрёдера, а также свойства решений, гарантирующих существование дробных итераций функции $f$. В работе [28] были получены условия вложимости $f \in \mathfrak{P}$ в однопараметрическую полугруппу в терминах решений уравнений Абеля и Шрёдера. Приведем два результата из этой работы.

Теорема 4. Пусть $f \in \mathfrak{P}$ отлична от дробно-линейного преобразования единичного круга $\mathbb{D}$ на себя, $f(0)=0 u f^{\prime}(0)=\gamma \neq 0$. Тогда $f$ вкладывается в однопараметрическую полугруппу в $\mathfrak{P}$ в том и только том случае, если существует решение F функиионального уравнения

$$
F \circ f(z)=\gamma F(z)
$$

представляющее собой голоморфную в $\mathbb{D}$ функцию, которая удовлетворяет условию

$$
\frac{z F^{\prime}(z)}{F(z)}=\frac{p(0)}{p(z)},
$$

где $p$ - голоморфная в $\mathbb{D}$ функция с положительной вещественной частью $u e^{-p(0)}=\gamma$.

Заметим, что в условиях этой теоремы $z=0$ является точкой Данжуа-Вольфа функции $f$, а в случае $f^{\prime}(0)=0$ функция $f$ не будет вложимой. Следующий результат касается случая граничной точки Данжуа-Вольфа [28].

Теорема 5. Пусть $f \in \mathfrak{P}$ отлична от дробно-линейного преобразования единичного круга $\mathbb{D}$ на себя и $z=1$ является ее точкой Данжуа-Вольфа. Тогда $f$ вкладьвается в однопараметрическую полугруппу в $\mathfrak{P}$ в том и только том случае, если существует решение $F$ функционального уравнения

$$
F \circ f(z)=F(z)+1,
$$


представляющее собой голоморфную в $\mathbb{D}$ функцию, которая удовлетворяет условию

$$
\operatorname{Re}\left\{(1-z)^{2} F^{\prime}(z)\right\}>0
$$

nрu $z \in \mathbb{D}$.

Допустим теперь, что $f \in \mathfrak{P}$ отлична от дробно-линейного преобразования единичного круга $\mathbb{D}$ на себя и имеет точку Данжуа-Вольфа $q \in \mathbb{D}$. Если $f^{\prime}(q)=$ $\gamma \neq 0$, то (см., например, [29]) существует предел

$$
\lim _{n \rightarrow \infty} \frac{f^{n}(z)-q}{\gamma^{n}}=K(z),
$$

который представляет собой голоморфную в $\mathbb{D}$ функцию, удовлетворяющую условиям $K(q)=0$ и $K^{\prime}(q)=1$. Эта функция является решением функционального уравнения Шрёдера

$$
K \circ f(z)=\gamma K(z),
$$

и ее называют функцией Кёнигса. Недавно в [24] было получено параметрическое представление класса функций Кёнигса (4), которые соответствуют вложимым функциям $f$.

Теорема 6. Для того чтобъ голоморфная в $\mathbb{D}$ функиия $K$ являласъ функцией Кёнигса (4) для некоторой вложимой в однопараметрическую полугрупnу в $\mathfrak{P}$ функиии $f$ с точкой Данжуа-Волъфа $q \in \mathbb{D}$, необходимо и достаточно, чтобы она допускала представление в виде

$$
K(z)=(z-q)\left(\frac{1-\bar{q} z}{1-|q|^{2}}\right)^{\sigma^{2}} \exp \left\{\left(1+\sigma^{2}\right) \int_{\mathbb{T}} \ln \frac{1-\varkappa q}{1-\varkappa z} d \mu(\varkappa)\right\}
$$

с некоторым $\sigma=e^{i \theta},-\pi / 2<\theta<\pi / 2$, и вероятностной мерой $\mu$ на единичной окружности $\mathbb{T}$. При этом под степенной функиией и логарифмом понимаются непрерывные ветви, которые принимают значения 1 и 0 соответственно при $z=q$.

Заметим также, что функция $K$, определяемая формулой (5), является однолистной и отображает $\mathbb{D}$ на $\theta$-спиральную область. При этом семейство функций

$$
f^{t}(z)=K^{-1}\left(e^{-\sigma t} K(z)\right),
$$

$t \geqslant 0$, определяет однопараметрическую полугруппу $t \mapsto f^{t}$ в $\mathfrak{P}$ с точкой Данжуа-Вольфа q. Таким образом, формула (5) определяет функции Кёнигса однопараметрических полугрупп в $\mathfrak{P}$ с точкой Данжуа-Вольфа $q$ из $\mathbb{D}$. Следующий результат выделяет те функции Кёнигса, которые отвечают однопараметрическим полугруппам с дополнительными неподвижными точками.

Теорема 7. Пусть $q \in \mathbb{D} u a_{1}, \ldots, a_{n}$ - попарно различные точки на единичной окружности $\mathbb{T}$. Для того чтобы голоморфная в $\mathbb{D}$ функиия $K$ представляла собой функиию Кёнигса однопараметрической полугруппь $t \mapsto f^{t}$ в $\mathfrak{P}$ с точкой Данжуа-Вольфа $q$ и неподвижными точками $a_{1}, \ldots, a_{n}$, в которых функиии $f^{t}, t>0$, имеют конечные угловые производные, необходимо 
и достаточно, чтобы для некоторых положительных чисел $\lambda_{1}, \ldots, \lambda_{n}$ u неотрицательного $\gamma$, удовлетворяющих условию $\lambda_{1}+\cdots+\lambda_{n}+\gamma=1, \sigma=e^{i \theta}$, $-\pi / 2<\theta<\pi / 2$, и вероятностной меры $\mu$ на $\mathbb{T}$ выполнялось равенство

$$
\begin{aligned}
K(z)=(z & -q)\left(\frac{1-\bar{q} z}{1-|q|^{2}}\right)^{\sigma^{2}} \prod_{k=1}^{n}\left(\frac{1-\bar{a}_{k} q}{1-\bar{a}_{k} z}\right)^{\lambda_{k}\left(1+\sigma^{2}\right)} \\
& \times \exp \left\{\gamma\left(1+\sigma^{2}\right) \int_{\mathbb{T}} \ln \frac{1-\varkappa q}{1-\varkappa z} d \mu(\varkappa)\right\},
\end{aligned}
$$

где под степенными функииями и логарифмом понимаются непрерывные ветви, которые принимают значения 1 и 0 соответственно при $z=q$.

ДокАЗАТЕЛьство. Пусть $t \mapsto f^{t}$ - однопараметрическая полугруппа в $\mathfrak{P}$ с точкой Данжуа-Вольфа $q \in \mathbb{D}$ и неподвижными точками $a_{1}, \ldots, a_{n} \in \mathbb{T}$, в которых функции $f^{t}$ имеют конечные угловые производные. Тогда ее инфинитезимальная образующая $v$ в силу теоремы 3 может быть представлена в виде

$$
v(z)=\frac{(q-z)(1-\bar{q} z)}{g(z)},
$$

где

$$
g(z)=\sum_{k=1}^{n} \alpha_{k} \frac{1+\bar{a}_{k} z}{1-\bar{a}_{k} z}+p(z),
$$

$\alpha_{k}>0, k=1, \ldots, n$, а $p$ - голоморфная в $\mathbb{D}$ с неотрицательной вещественной частью функция. Если $K$ - функция Кёнигса, отвечающая этой однопараметрической полугруппе, то при всех $t \geqslant 0$ будут выполняться равенства

$$
K \circ f^{t}(z)=\left(f^{t}\right)^{\prime}(q) K(z) .
$$

Дифференцируя эти равенства по $t$, получаем следующее соотношение:

$$
K^{\prime}\left(f^{t}(z)\right) v\left(f^{t}(z)\right)=v^{\prime}(q) e^{t v^{\prime}(q)} K(z) .
$$

Здесь мы воспользовались равенством $\left(f^{t}\right)^{\prime}(q)=e^{v^{\prime}(q) t}$, которое получается в результате интегрирования дифференциального уравнения

$$
\frac{d}{d t}\left(f^{t}\right)^{\prime}(q)=v^{\prime}(q)\left(f^{t}\right)^{\prime}(q)
$$

с начальным условием $\left.\left(f^{t}\right)^{\prime}(q)\right|_{t=0}=1$. Полагая в полученном выше соотношении $t=0$, приходим к равенству

$$
K^{\prime}(z) v(z)=v^{\prime}(q) K(z)
$$

Равенство (7) можно рассматривать как дифференциальное уравнение для функции Кёнигса $K$, которое с начальными условиями $K(q)=0, K^{\prime}(q)=1$ имеет единственное решение.

Определим теперь функцию $K$ формулой (6), в которой параметры выберем следующим образом. Числа $\lambda_{k}, k=1, \ldots, n$, и $\gamma$ определим равенствами

$$
\lambda_{k}=\frac{\alpha_{k}}{\operatorname{Re} g(q)} \frac{1-|q|^{2}}{\left|1-\bar{a}_{k} q\right|^{2}}, \quad \gamma=1-\sum_{k=1}^{n} \lambda_{k} .
$$


Заметим, что

$$
\sum_{k=1}^{n} \lambda_{k}=\frac{1}{\operatorname{Re} g(q)} \operatorname{Re}\left\{\sum_{k=1}^{n} \alpha_{k} \frac{1+\bar{a}_{k} q}{1-\bar{a}_{k} q}\right\}=\frac{\operatorname{Re}\{g(q)-p(q)\}}{\operatorname{Re} g(q)}=1-\frac{\operatorname{Re} p(q)}{\operatorname{Re} g(q)}
$$

и, следовательно,

$$
\gamma=\frac{\operatorname{Re} p(q)}{\operatorname{Re} g(q)} \geqslant 0
$$

Далее, в силу формулы Рисса-Герглотца функцию $p$ можно представить в виде

$$
p(z)=\lambda \int_{\mathbb{T}} \frac{1+\varkappa z}{1-\varkappa z} d \nu(\varkappa)+i \beta
$$

где $\nu$ - вероятностная мера на $\mathbb{T}, \lambda=\operatorname{Re} p(0) \geqslant 0, \beta=\operatorname{Im} p(0)$. В качестве вероятностной меры $\mu$ в формуле (6) возьмем преобразованную из $\nu$ меру

$$
d \mu(\varkappa)=\frac{\operatorname{Re} p(0)}{\operatorname{Re} p(q)} \frac{1-|q|^{2}}{|1-\varkappa q|^{2}} d \nu(\varkappa) .
$$

Очевидно, что $\mu$ также является вероятностной мерой на $\mathbb{T}$. Наконец, положим $\sigma^{2}=\overline{g(q)} / g(q)$.

Таким образом, для завершения доказательства необходимости представления функции Кёнигса в виде (6) достаточно показать, что с определенными выше параметрами функция $K$ удовлетворяет равенству (7). Дифференцируя равенство (6), получаем

$$
\begin{aligned}
\frac{K^{\prime}(z)}{K(z)}= & \frac{1}{z-q}-\sigma^{2} \frac{\bar{q}}{1-\bar{q} z}+\left(1+\sigma^{2}\right)\left[\sum_{k=1}^{n} \lambda_{k} \frac{\bar{a}_{k}}{1-\bar{a}_{k} z}+\gamma \int_{\mathbb{T}} \frac{\varkappa}{1-\varkappa z} d \mu(\varkappa)\right] \\
= & \frac{1-|q|^{2}}{(z-q)(1-\bar{q} z)}+\left(1+\sigma^{2}\right)\left[\sum_{k=1}^{n} \lambda_{k}\left(\frac{\bar{a}_{k}}{1-\bar{a}_{k} z}-\frac{\bar{q}}{1-\bar{q} z}\right)\right. \\
& \left.\quad+\gamma \int_{\mathbb{T}}\left(\frac{\varkappa}{1-\varkappa z}-\frac{\bar{q}}{1-\bar{q} z}\right) d \mu(\varkappa)\right] .
\end{aligned}
$$

Отсюда следует равенство

$$
\begin{aligned}
\frac{(z-q)(1-\bar{q} z) K^{\prime}(z)}{\left(1-|q|^{2}\right) K(z)}=1 & +\left(1+\sigma^{2}\right) \sum_{k=1}^{n} \lambda_{k} \frac{\left(\bar{a}_{k}-\bar{q}\right)(z-q)}{\left(1-\bar{a}_{k} z\right)\left(1-|q|^{2}\right)} \\
& +\left(1+\sigma^{2}\right) \gamma \int_{\mathbb{T}} \frac{(\varkappa-\bar{q})(z-q)}{(1-\varkappa z)\left(1-|q|^{2}\right)} d \mu(\varkappa) .
\end{aligned}
$$

Учитывая выбор параметров $\sigma, \gamma$ и $\lambda_{k}$, это равенство можно преобразовать следующим образом:

$$
\begin{aligned}
\frac{(z-q)(1-\bar{q} z) K^{\prime}(z)}{\left(1-|q|^{2}\right) K(z)}=1 & +\frac{2}{g(q)} \sum_{k=1}^{n} \alpha_{k} \frac{\left(\bar{a}_{k}-\bar{q}\right)(z-q)}{\left(1-\bar{a}_{k} z\right)\left|1-\bar{a}_{k} q\right|^{2}} \\
& +\frac{2 \operatorname{Re} p(q)}{g(q)} \int_{\mathbb{T}} \frac{(\varkappa-\bar{q})(z-q)}{\left(1-|q|^{2}\right)(1-\varkappa z)} d \mu(\varkappa) .
\end{aligned}
$$

Выполняя замену меры $\mu$ на $\nu$ и учитывая связь между функциями $p$ и $g$, получаем

$$
\begin{gathered}
\frac{g(q)(z-q)(1-\bar{q} z) K^{\prime}(z)}{\left(1-|q|^{2}\right) K(z)}=p(q)+\sum_{k=1}^{n} \alpha_{k}\left(\frac{2\left(\bar{a}_{k}-\bar{q}\right)(z-q)}{\left(1-\bar{a}_{k} z\right)\left|1-\bar{a}_{k} q\right|^{2}}+\frac{1+\bar{a}_{k} q}{1-\bar{a}_{k} q}\right) \\
+2 \lambda \int_{\mathbb{T}} \frac{(\varkappa-\bar{q})(z-q)}{|1-\varkappa|^{2}(1-\varkappa z)} d \nu(\varkappa) .
\end{gathered}
$$


Далее, поскольку

а также

$$
\frac{2\left(\bar{a}_{k}-\bar{q}\right)(z-q)}{\left(1-\bar{a}_{k} z\right)\left|1-\bar{a}_{k} q\right|^{2}}+\frac{1+\bar{a}_{k} q}{1-\bar{a}_{k} q}=\frac{1+\bar{a}_{k} z}{1-\bar{a}_{k} z}
$$

$$
p(q)+2 \lambda \int_{\mathbb{T}} \frac{(\varkappa-\bar{q})(z-q)}{|1-\varkappa|^{2}(1-\varkappa z)} d \nu(\varkappa)=\lambda \int_{\mathbb{T}} \frac{1+\varkappa z}{1-\varkappa z} d \nu(\varkappa)+i \beta=p(z),
$$

приходим к следующему равенству:

$$
\frac{K^{\prime}(z)}{K(z)}=\frac{\left(1-|q|^{2}\right) g(z)}{g(q)(z-q)(1-\bar{q} z)}=\frac{v^{\prime}(q)}{v(z)} .
$$

Это означает выполнение равенства (7), и необходимость доказана.

Достаточность условия представления функции Кёнигса формулой (6) была установлена в [24] (см. предложение 2). Теорема доказана.

В случае граничной точки Данжуа-Вольфа $q \in \mathbb{T}$ определение функции Кёнигса, аналогичное (4), затрудняется тем, что соответствующая предельная функция может оказаться тождественно постоянной (см., например, [29]). В работах [30], [31] изучались другие способы нормировки последовательности итераций для получения в пределе не тождественно постоянной функции в случае граничной точки Данжуа-Вольфа. При этом, как правило, предельная функция является решением функционального уравнения Абеля. В работе [24] было показано, что каждой однопараметрической полугруппе $t \mapsto f^{t}$ в $\mathfrak{P}$ с точкой Данжуа-Вольфа $q \in \mathbb{T}$ соответствует единственная функция $F$, которая является решением уравнения

$$
F \circ f^{t}(z)=F(z)+t
$$

при всех $t \geqslant 0$ и удовлетворяет условию $F(0)=0$. Эту функцию естественно называть функиией Кёнигса однопараметрической полугруппь $t \mapsto f^{t}$ в случае граничной точки Данжуа-Вольфа. В работе [24] получено параметрическое представление класса функций Кёнигса, отвечающих однопараметрическим полугруппам с заданной точкой Данжуа-Вольфа $q \in \mathbb{T}$.

ТеОрема 8. Для того чтобы голоморфная в $\mathbb{D}$ функиия $F$ являлась функцией Кёнигса однопараметрической полугруппь $t \mapsto f^{t}$ в $\mathfrak{P}$ с точкой Данжуа-Вольфа $q \in \mathbb{T}$, необходимо и достаточно, чтобы она допускала представление в виде

$$
\begin{aligned}
F(z)= & i \beta \frac{\bar{q} z}{1-\bar{q} z}+\lambda_{1} \frac{\bar{q} z}{(1-\bar{q} z)^{2}} \\
& +\lambda_{2} \int_{\mathbb{T} \backslash\{\bar{q}\}}\left(\ln \frac{1-\varkappa z}{1-\bar{q} z}+i \operatorname{Im}\{\varkappa q\} \frac{\bar{q} z}{1-\bar{q} z}\right) \frac{d \mu(\varkappa)}{1-\operatorname{Re}\{\varkappa q\}}
\end{aligned}
$$

с некоторыми $\beta \in \mathbb{R}, \lambda_{1}, \lambda_{2} \geqslant 0$ и вероятностной мерой $\mu$ на $\mathbb{T} \backslash\{\bar{q}\}$. При этом под логарифмом понимается непрерывная ветвъ, обращающаяся в нуль при $z=0$.

Заметим, что сходимость интеграла в определении функции Кёнигса обеспечивается тем, что

$$
\lim _{\varkappa \rightarrow \bar{q}}\left\{\frac{1}{1-\operatorname{Re}\{\varkappa q\}}\left(\ln \frac{1-\varkappa z}{1-\bar{q} z}+i \operatorname{Im}\{\varkappa q\} \frac{\bar{q} z}{1-\bar{q} z}\right)\right\}=\frac{\bar{q} z}{(1-\bar{q} z)^{2}} .
$$


Вопрос о выделении тех функций Кёнигса $F$, которые отвечают однопараметрическим полугруппам в $\mathfrak{P}$ с заданным множеством неподвижных точек, peшается в следующей теореме.

Теорема 9. Пустъ $q, a_{1}, \ldots, a_{n}$ - попарно различные точки на единичной окружности $\mathbb{T}$. Для того чтобы голоморфная в $\mathbb{D}$ функиця $F$ являлась функичей Кёнигса однопараметрической полугруппь $t \mapsto f^{t}$ в $\mathfrak{P}$ с точкой Данжуа-Вольфа q и неподвижными точками $a_{1}, \ldots, a_{n}$, в которьх функиии $f^{t}$, $t>0$, имеют конечные угловые производные, необходимо и достаточно, чтобы для некоторых положительных чисел $\lambda_{1}, \ldots, \lambda_{n}$, неотрицательных $\gamma_{1}, \gamma_{2}$, $\beta \in \mathbb{R}$ и вероятностной меры $\mu$ на $\mathbb{T} \backslash\{\bar{q}\}$ выполнялось равенство

$$
\begin{aligned}
F(z)=i \beta & \frac{\bar{q} z}{1-\bar{q} z}+\sum_{k=1}^{n} \lambda_{k} \ln \frac{1-\bar{a}_{k} z}{1-\bar{q} z}+\gamma_{1} \frac{\bar{q} z}{(1-\bar{q} z)^{2}} \\
& +\gamma_{2} \int_{\mathbb{T} \backslash\{\bar{q}\}}\left(\ln \frac{1-\varkappa z}{1-\bar{q} z}+i \operatorname{Im}\{\varkappa q\} \frac{\bar{q} z}{1-\bar{q} z}\right) \frac{d \mu(\varkappa)}{1-\operatorname{Re}\{\varkappa q\}},
\end{aligned}
$$

где под логарифмами понимаются непрерывные ветви, обращающиеся в нуль при $z=0$.

ДокАЗАТЕЛьство. Пусть $t \mapsto f^{t}$ - однопараметрическая полугруппа в $\mathfrak{P}$ с точкой Данжуа-Вольфа $q \in \mathbb{T}$ и неподвижными точками $a_{1}, \ldots, a_{n}$, в которых функции $f^{t}$ имеют конечные угловые производные. Поскольку $|q|=1$, то инфинитезимальную образующую $v$ этой однопараметрической полугруппы можно представить в виде

где

$$
v(z)=\frac{q(1-\bar{q} z)^{2}}{g(z)}
$$

$$
g(z)=\sum_{k=1}^{n} \alpha_{k} \frac{1+\bar{a}_{k} z}{1-\bar{a}_{k} z}+p(z)
$$

$\alpha_{k}>0, k=1, \ldots, n$, а $p$ - голоморфная в $\mathbb{D}$ функция с неотрицательной вещественной частью. Пусть $F$ - функция Кёнигса однопараметрической полугруппы $t \mapsto f^{t}$, т. е. для всех $t \geqslant 0$ выполняется равенство

$$
F \circ f^{t}(z)=F(z)+t .
$$

Дифференцируя это равенство по $t$ и полагая $t=0$, получаем

$$
F^{\prime}(z)=\frac{1}{v(z)}=\frac{g(z)}{q(1-\bar{q} z)^{2}} .
$$

Отсюда в силу односвязности единичного круга $\mathbb{D}$ функция $F$ определяется однозначно с учетом условия $F(0)=0$.

Определим теперь функцию $F$ формулой (8), в которой параметры выберем следующим образом. В силу теоремы Рисса-Герглотца функция $p$ представима в виде

$$
p(z)=\int_{\mathbb{T}} \frac{1+\varkappa z}{1-\varkappa z} d \nu(\varkappa)+i \gamma,
$$

где $\gamma \in \mathbb{R}$, а $\nu$ - конечная неотрицательная борелевская мера на $\mathbb{T}$. Положим

$$
\lambda_{k}=\frac{\alpha_{k}}{1-\operatorname{Re}\left\{\bar{a}_{k} q\right\}}, \quad \beta=\gamma+\sum_{k=1}^{n} \lambda_{k} \operatorname{Im}\left\{\bar{a}_{k} q\right\}, \quad \gamma_{1}=\nu(\{\bar{q}\}), \quad \gamma_{2}=\nu(\mathbb{T})-\gamma_{1} .
$$


Если $\gamma_{2}=0$, то определение меры $\mu$ не имеет значения. В случае $\gamma_{2}>0$ вероятностную меру $\mu$ на $\mathbb{T} \backslash\{\bar{q}\}$ определим равенством

$$
d \mu(\varkappa)=\frac{1}{\gamma_{2}} d \nu(\varkappa)
$$

Покажем, что определенная с этими параметрами функция $F$ удовлетворяет условию $F^{\prime}(z)=1 / v(z)$. Этим будет доказана необходимость представления функции Кёнигса формулой (8). Непосредственные вычисления показывают, что

$$
\begin{aligned}
F^{\prime}(z)= & i \beta \frac{\bar{q}}{(1-\bar{q} z)^{2}}+\sum_{k=1}^{n} \lambda_{k} \frac{\bar{q}-\bar{a}_{k}}{(1-\bar{q} z)\left(1-\bar{a}_{k} z\right)}+\gamma_{1} \frac{\bar{q}(1+\bar{q} z)}{(1-\bar{q} z)^{3}} \\
& +\gamma_{2} \int_{\mathbb{T} \backslash\{\bar{q}\}}\left(\frac{\bar{q}-\varkappa}{(1-\bar{q} z)(1-\varkappa z)}+i \operatorname{Im}\{\varkappa q\} \frac{\bar{q}}{(1-\bar{q} z)^{2}}\right) \frac{d \mu(\varkappa)}{1-\operatorname{Re}\{\varkappa q\}} \\
= & \frac{\bar{q}}{(1-\bar{q} z)^{2}}\left[i \beta+\sum_{k=1}^{n} \lambda_{k} \frac{\left(1-\bar{a}_{k} q\right)(1-\bar{q} z)}{1-\bar{a}_{k} z}+\gamma_{1} \frac{1+\bar{q} z}{1-\bar{q} z}\right. \\
& \left.+\gamma_{2} \int_{\mathbb{T} \backslash\{\bar{q}\}}\left(\frac{(1-\varkappa q)(1-\bar{q} z)}{1-\varkappa z}+i \operatorname{Im}\{\varkappa q\}\right) \frac{d \mu(\varkappa)}{1-\operatorname{Re}\{\varkappa q\}}\right] .
\end{aligned}
$$

Используя равенства

$$
\begin{aligned}
& \frac{\left(1-\bar{a}_{k} q\right)(1-\bar{q} z)}{1-\bar{a}_{k} z}=\left(1-\operatorname{Re}\left\{\bar{a}_{k} q\right\}\right) \frac{1+\bar{a}_{k} z}{1-\bar{a}_{k} z}-i \operatorname{Im}\left\{\bar{a}_{k} q\right\}, \\
& \frac{(1-\varkappa q)(1-\bar{q} z)}{1-\varkappa z}=(1-\operatorname{Re}\{\varkappa q\}) \frac{1+\varkappa z}{1-\varkappa z}-i \operatorname{Im}\{\varkappa q\},
\end{aligned}
$$

полученное представление для $F^{\prime}(z)$ можно переписать в виде

$$
\begin{aligned}
F^{\prime}(z)= & \frac{\bar{q}}{(1-\bar{q} z)^{2}}\left[i \beta+\sum_{k=1}^{n} \lambda_{k}\left(1-\operatorname{Re}\left\{\bar{a}_{k} q\right\}\right) \frac{1+\bar{a}_{k} z}{1-\bar{a}_{k} z}\right. \\
& \left.-i \sum_{k=1}^{n} \lambda_{k} \operatorname{Im}\left\{\bar{a}_{k} q\right\}+\gamma_{1} \frac{1+\bar{q} z}{1-\bar{q} z}+\gamma_{2} \int_{\mathbb{T} \backslash\{\bar{q}\}} \frac{1+\varkappa z}{1-\varkappa z} d \mu(\varkappa)\right] .
\end{aligned}
$$

Учитывая теперь выбор параметров, получаем

$$
\begin{aligned}
F^{\prime}(z) & =\frac{\bar{q}}{(1-\bar{q} z)^{2}}\left[i \gamma+\sum_{k=1}^{n} \alpha_{k} \frac{1+\bar{a}_{k} z}{1-\bar{a}_{k} z}+\int_{\mathbb{T}} \frac{1+\varkappa z}{1-\varkappa z} d \nu(\varkappa)\right] \\
& =\frac{\bar{q}}{(1-\bar{q} z)^{2}}\left[\sum_{k=1}^{n} \alpha_{k} \frac{1+\bar{a}_{k} z}{1-\bar{a}_{k} z}+p(z)\right]=\frac{1}{v(z)} .
\end{aligned}
$$

Необходимость, таким образом, доказана.

Достаточность условия представления функции Кёнигса формулой (8) была установлена в [24] (см. предложение 3). Теорема доказана.

Связь между наличием неподвижных точек у однопараметрической полугруппы и поведением функции Кёнигса изучалась многими авторами. Отметим в этой связи работу [32]. 


\section{4. Эволюционные семейства и эволюционное уравнение}

С точки зрения динамики семейство функций $f^{t}, t \geqslant 0$, однопараметрической полугруппы $t \mapsto f^{t}$ в $\mathfrak{P}$ образует полупоток. При этом, если $v-$ инфинитезимальная образующая этой однопараметрической полугруппы, то полупоток $f^{t}, t \geqslant 0$, порожден векторным полем $v(z)$, поскольку $w=f^{t}(z)$ - решение автономного дифференциального уравнения

$$
\frac{d w}{d t}=v(w)
$$

с начальным условием $\left.w\right|_{t=0}=z$. Другими словами, отображение $t \mapsto f^{t}(z)$ представляет собой интегральную кривую дифференциального уравнения (9), а отображение $z \mapsto f^{t}(z), z \in \mathbb{D}$, осуществляет сдвиг вдоль интегральных кривых этого уравнения. Как видно из результатов предыдущего раздела, далеко не каждую функцию $f \in \mathfrak{P}$ можно вложить в однопараметрическую полугруппу. Естественная замена автономного уравнения (9) на неавтономное

$$
\frac{d w}{d t}=V(w, t)
$$

где при каждом $t>0$ функция $V(\cdot, t)$ представляет собой инфинитезимальное преобразование полугруппы $\mathfrak{P}$, должна привести к более широкому классу вложимых функций $f$. При этом нужно перейти от однопараметрической полугруппы к более общей конструкции и конкретизировать условия на правую часть уравнения (10) так, чтобы были гарантированы существование и единственность решения задачи Коши для этого уравнения.

ОПредЕлЕНиЕ 1. Двупараметрическое семейство $\left\{w_{t, s}: 0 \leqslant s \leqslant t \leqslant T\right\}$ полугруппы $\mathfrak{P}$ будем называть эволюиионным семейством в $\mathfrak{P}$, если выполняются следующие условия:

(i) $w_{t, s}(z)=w_{t, \tau} \circ w_{\tau, s}(z)$ при $0 \leqslant s \leqslant \tau \leqslant t \leqslant T$,

(ii) $w_{t, s}(z) \rightarrow z$ локально равномерно в $\mathbb{D}$ при $(t-s) \rightarrow 0$.

Заметим, что если $t \mapsto f^{t}$ - однопараметрическая полугруппа в $\mathfrak{P}$, то

$$
\left\{w_{t, s}=f^{t-s}: 0 \leqslant s \leqslant t \leqslant T\right\}
$$

является эволюционным семейством в $\mathfrak{P}$. При этом в отличие от случая однопараметрической полугруппы вопрос дифференцируемости эволюционного семейства решается гораздо сложнее. Впервые эти вопросы для полугруппы $\mathfrak{L}$ голоморфных и однолистных в $\mathbb{D}$ функций $f$, удовлетворяющих условиям $f(0)=0, f^{\prime}(0)>0$ и $|f(z)|<1$ при $z \in \mathbb{D}$, рассмотрел Лёвнер [3]. Очевидно, что функция $f$ из $\mathfrak{L}$ имеет в качестве точки Данжуа-Вольфа начало координат, а инфинитезимальные преобразования полугруппы $\mathfrak{L}$ описываются формулой $v(z)=-z p(z)$, где $p$ - голоморфная в $\mathbb{D}$ функция с неотрицательной вещественной частью. Таким образом, эволюционное уравнение (10) для полугруппы $\mathfrak{L}$ принимает вид

$$
\frac{d w}{d t}=-w P(w, t)
$$

где $P(\cdot, t), 0 \leqslant t \leqslant T,-$ семейство голоморфных в $\mathbb{D}$ функций с неотрицательной вещественной частью. 
Пусть теперь $\left\{w_{t, s}: 0 \leqslant s \leqslant t \leqslant T\right\}-$ эволюционное семейство в полугруппе $\mathfrak{L}$. Тогда семейство функций $g(z, t)=w_{T, t}(z), 0 \leqslant t \leqslant T$, обладает тем свойством, что

$$
g\left(z, t^{\prime}\right)=w_{T, t^{\prime}}(z)=w_{T, t^{\prime \prime}} \circ w_{t^{\prime \prime}, t^{\prime}}(z)=g\left(w_{t^{\prime \prime}, t^{\prime}}(z), t^{\prime \prime}\right)
$$

при $0 \leqslant t^{\prime} \leqslant t^{\prime \prime} \leqslant T$, т. е. области $g(\mathbb{D}, t), 0 \leqslant t \leqslant T$, расширяются при возрастании $t$ и $g(\mathbb{D}, T)=\mathbb{D}$. Кроме того, в силу теоремы Каратеодори и условия (ii) это семейство областей зависит от $t$ непрерывно в смысле сходимости к ядру. Обратно, по семейству функций $g(z, t), 0 \leqslant t \leqslant T$, отображающих единичный круг $\mathbb{D}$ на семейство областей с перечисленными свойствами и нормированных условиями $g(0, t)=0, g^{\prime}(0, t)>0$, однозначно восстанавливается эволюционное семейство $\left\{w_{t, s}: 0 \leqslant s \leqslant t \leqslant T\right\}$ посредством равенства

$$
w_{t, s}(z)=g^{-1}(g(z, s), t) .
$$

Сделанное замечание позволяет конструировать эволюционные семейства в $\mathfrak{L}$. Случай, когда области $g(\mathbb{D}, t)$ получаются удалением из $\mathbb{D}$ разреза вдоль жордановой дуги, был детально изучен в работе Лёвнера [3]. При этом функции $g(z, t)$ нормировались условием

$$
\left.\frac{d}{d z} g(z, t)\right|_{z=0}=g^{\prime}(0, t)=e^{t-T},
$$

$0 \leqslant t \leqslant T=\ln (1 / \beta)$, где $\beta=g^{\prime}(0,0)$, а соответствующее эволюционное семейство удовлетворяло условию

$$
w_{t, s}^{\prime}(0)=e^{s-t},
$$

$0 \leqslant s \leqslant t \leqslant T$. При такой нормировке вопрос дифференцируемости по $t$ эволюционного семейства и функции $g(z, t)$ решается легко с использованием следующей леммы Лёвнера [3].

Лемма 1. Пусть $f \in \mathfrak{L} u f^{\prime}(0)=\beta$. Тогда для всех $z \in \mathbb{D}$ выполняется неравенство

$$
|f(z)-z| \leqslant(1-\beta)|z| \frac{1+|z|}{1-|z|} .
$$

Эта лемма показывает, что окрестность тождественного преобразования (относительно топологии локально равномерной сходимости в $\mathbb{D}$ ) в полугруппе $\mathfrak{L}$ описывается в терминах величины $f^{\prime}(0)$. Кроме того, Лёвнер показал, что семейство функций $g(z, t), 0 \leqslant t \leqslant T$, удовлетворяет дифференциальному уравнению

$$
\frac{\partial}{\partial t} g(z, t)=z P(z, t) \frac{\partial}{\partial z} g(z, t),
$$

в котором функция $P$ имеет вид

$$
P(z, t)=\frac{1+\varkappa(t) z}{1-\varkappa(t) z},
$$

где $\varkappa(t)$ - непрерывная унимодулярная (т. е. $|\varkappa(t)| \equiv 1)$ функция на $[0, T]$. Эволюционное семейство $\left\{w_{t, s}: 0 \leqslant s \leqslant t \leqslant T\right\}$ при этом воспроизводится эволюционным уравнением (11) с той же функцией $P(z, t)$. Более точно, $w=w_{t, s}(z)-$ решение уравнения (11) с начальным условием $\left.w\right|_{t=s}=z, 0 \leqslant s \leqslant t \leqslant T$. 
Позже в работах Куфарева [33] и Поммеренке [34], [35] вопрос дифференцируемости семейств конформных отображений $g(z, t), t \geqslant 0$, единичного круга $\mathbb{D}$ на расширяющиеся области изучался в более общей постановке. В частности, было установлено, что соответствующим образом нормированное семейство функций $g(z, t), t \geqslant 0$, удовлетворяет уравнению (12) с функцией $P(z, t)$, которая измерима по $t$, голоморфна по $z$ в $\mathbb{D}$ и имеет неотрицательную вещественную часть. В связи с этим в литературе часто уравнения (11) и (12) с функцией $P(z, t)$ вида (13) связывают с именем Лёвнера, а в случае, когда $P(z, t)$ измерима по $t$, голоморфна по $z$ в $\mathbb{D}$ и имеет неотрицательную вещественную часть, (11) и (12) называют уравнениями Лёвнера-Куфарева. В работе [36] было показано, что семейство функций $g(z, t)$ и соответствующее эволюционное семейство, которые воспроизводятся посредством уравнений (11), (12) с функцией $P(z, t)$ вида (13), не обязаны состоять из отображений единичного круга на области, получаемые стиранием разреза. Этот вопрос вновь привлек внимание (см., например, [37]-[39]) в связи с развитием стохастической эволюции Лёвнера, известной также в литературе как SLE (см. [40] и цитированную там литературу).

Следующий результат дает достаточно общую теорему существования для эволюционного уравнения (11), см. [41].

Теорема 10. Пусть на $\mathbb{D} \times[0, T], T>0$, определена комплекснозначная с неотрицательной вещественной частью функиия $P(z, t)$, которая голоморфна по $z$, измерима по $t$ и такова, что $|P(0, t)|$ - суммируемая на $[0, T]$ функиия. Тогда для любых $z \in \mathbb{D} u s \in[0, T)$ существует единственное абсолютно непрерывное на $[s, T]$ решение $w=w(t, z, s ; P)$ уравнения $(11)$ с начальным условием $\left.w\right|_{t=s}=z$. При этом $w_{t, s}^{P}: z \mapsto w(t, z, s ; P)$ осуществляет конформное отображсение единичного круга $\mathbb{D}$ в себя, оставляя неподвижным начало координат.

Таким образом, функция $P(z, t)$, удовлетворяющая условиям теоремы 10 , определяет эволюционное семейство $\left\{w_{t, s}^{P}: 0 \leqslant s \leqslant t \leqslant T\right\}$ в $\mathfrak{P}$. Если дополнительно потребовать, чтобы выполнялось условие $\operatorname{Im} P(0, t) \equiv 0$, то получим эволюционное семейство в полугруппе $\mathfrak{L}$. Лёвнер показал, что любая $f \in \mathfrak{L}$ может быть аппроксимирована в топологии локально равномерной сходимости голоморфных в $\mathbb{D}$ функций преобразованиями $w_{T, 0}^{P}, T \geqslant 0$, с функциями $P(z, t)$ вида (13). Благодаря исследованиям Куфарева [33], Поммеренке [34] и Гутлянского [42] этот результат получил завершенную форму: каждое отображение $f$ из $\mathfrak{L}$ может быть представлено в виде $f=w_{T, 0}^{P}$ с некоторыми $T \geqslant 0$ и функцией $P(z, t)$, которая измерима по $t$ на $[0, T]$, голоморфна по $z$ в $\mathbb{D}$, имеет неотрицательную вещественную часть и такова, что $P(0, t) \equiv 1$. Другими словами, всякую функцию $f$ полугруппы $\mathfrak{L}$ можно вложить в некоторое эволюционное семейство полугруппы $\mathfrak{L}$, которое воспроизводится эволюционным уравнением. При этом $P(z, t)$ из формулировки теоремы 10 можно назвать инфинитезималъной производящей функиией эволюционного семейства $\left\{w_{t, s}^{P}: 0 \leqslant s \leqslant t \leqslant T\right\}$.

В работах [43], [44] (см. также [41]) был решен вопрос о топологии в пространстве инфинитезимальных производящих функций, которая соответству- 
ет локально равномерной в $\mathbb{D}$ сходимости эволюционных семейств. Приведем соответствующие определения и некоторые результаты.

Пусть $D$ - область в конечной комплексной плоскости $\mathbb{C}$ и $\mathscr{H}(D)$ - линейное пространство всех голоморфных в $D$ функций, наделенное топологией локально равномерной в $D$ сходимости. В действительности $\mathscr{H}(D)$ является метризуемым полным локально выпуклым пространством, или пространством Фреше. Поэтому многие основные результаты о компактных выпуклых множествах (см., например, [45], [46]) справедливы в пространстве $\mathscr{H}(D)$. В частности, если $\mathscr{K} \subset \mathscr{H}(D)$ - компактное подмножество, то компактна также его замкнутая выпуклая оболочка со $\mathscr{K}$ и, в силу теоремы Крейна-Мильмана,

$$
\overline{\operatorname{co}} \mathscr{K}=\overline{\operatorname{co}} \operatorname{ext} \mathscr{K}, \quad \operatorname{ext} \overline{\operatorname{co}} \mathscr{K} \subseteq \operatorname{ext} \mathscr{K} \subseteq \mathscr{K},
$$

где ext $\mathscr{K}$ - совокупность крайних точек множества $\mathscr{K}$.

Далее, пусть $I=[\alpha, \beta] \subset \mathbb{R}$ и $\mathfrak{F}(D, I)$ - совокупность комплекснозначных на $D \times I$ функций $F(z, t)$, голоморфных по $z$, измеримых по $t$ и таких, что для любого компактного подмножества $\Delta$ области $D$ функция

$$
M(t ; F, \Delta)=\sup \{|F(z, t)|: z \in \Delta\}
$$

является суммируемой на $I$. Очевидно, что $\mathfrak{F}(D, I)$ представляет собой линейное пространство над полем комплексных чисел. Кроме того, стандартные рассуждения с использованием интегральной формулы Коши показывают, что операция дифференцирования по $z$ преобразует пространство $\mathfrak{F}(D, I)$ в себя, а интеграл $\int_{I} F(z, t) d t$ представляет собой голоморфную в $D$ функцию.

ОПРЕДЕЛЕНИЕ 2. Пусть $F, F_{n} \in \mathfrak{F}(D, I), n=1,2, \ldots$. Будем говорить, что последовательность $\left\{F_{n}\right\}$ слабо сходится в $\mathfrak{F}(D, I)$ к функции $F$, если для любой ограниченной измеримой на $I$ функции $\eta(t)$

$$
\int_{I} \eta(t) F_{n}(z, t) d t \rightarrow \int_{I} \eta(t) F(z, t) d t
$$

локально равномерно в $D$ при $n \rightarrow \infty$.

Значение этого определения для эволюционных семейств раскрывается в следующем результате [43], [41].

Теорема 11. Пусть на $\mathbb{D} \times[0, T], T>0$, определены комплекснозначные функции $P_{n}(z, t), n=1,2, \ldots$, с неотрицательной вещественной частью, которые голоморфны по $z$, измеримы по $t$ и таковы, что

$$
\left|P_{n}(z, t)\right| \leqslant M(t)
$$

для почти всех $t \in[0, T]$ и $n=1,2, \ldots$, где $M(t)$ - суммируемая на $[0, T]$ бункиия. Тогда $P_{n} \in \mathfrak{F}(\mathbb{D},[0, T]), n=1,2, \ldots$, и эквивалентны следующие утверждения:

(а) последовательность $\left\{P_{n}\right\}$ слабо сходится в $\mathfrak{F}(\mathbb{D},[0, T])$ к функиии $P \in$ $\mathfrak{F}(\mathbb{D},[0, T]) ;$

(b) бункиия $P(z, t)$ удовлетворяет тем же требованиям, что и функции $P_{n}, n=1,2, \ldots$, и $w_{t, s}^{P_{n}}(z) \rightarrow w_{t, s}^{P}(z)$ локально равномерно в $\mathbb{D} n р и$ $n \rightarrow \infty$ для всех $s, t \in[0, T], s \leqslant t$. 
Отметим некоторые важные свойства слабой сходимости в $\mathfrak{F}(D, I)$. В частности, имеет место следующий принцип компактности [43], [41].

Tеорема 12. Пусть $F_{n} \in \mathfrak{F}(D, I), n=1,2, \ldots$, и для каждого компактного подмножества $\Delta \subset D$ существует суммируемая на $I$ функиия $M_{\Delta}(t)$ такая, чmo

$$
\left|F_{n}(z, t)\right| \leqslant M_{\Delta}(t)
$$

для почти всех $t \in I, z \in \Delta$ u $n=1,2, \ldots$. Тогда из последователвности $\left\{F_{n}\right\}$ можно выделить подпоследовательность, слабо сходящуюся в $\mathfrak{F}(D, I)$. Кроме того, предельная функиия $F(z, t)$ любой слабо сходящейся подпоследовательности из $\left\{F_{n}\right\}$ удовлетворяет условию

$$
F(\cdot, t) \in \overline{\mathrm{co}}\left\{F_{n}(\cdot, t): n=1,2, \ldots\right\}
$$

для почти всех $t \in I$.

Пусть $\mathscr{K} \subset \mathscr{H}(D)$ - некоторое множество голоморфных в $D$ функций. Мы будем говорить, что функция $F(z, t)$ из $\mathfrak{F}(D, I)$ является $\mathscr{K}$-значной, если $F(\cdot, t) \in \mathscr{K}$ для почти всех $t \in I$. В связи с этим понятием можно сформулировать утверждение, непосредственно вытекающее из предыдущей теоремы.

СлеДСтвиЕ 1. Пусть $\mathscr{K}$ - компактное выпуклое подмножество пространства $\mathscr{H}(D)$. Тогда множество $\mathscr{K}$-значных функций компактно (более точно, секвенииально компактно) относительно слабой сходимости в $\mathfrak{F}(D, I)$.

Будем говорить, что функция $F(z, t)$ из $\mathfrak{F}(D, I)$ является ступенчатой, если существуют конечное разбиение $\alpha=t_{0}<t_{1}<\cdots<t_{n}=\beta$ отрезка $I=[\alpha, \beta]$ и набор функций $f_{1}, \ldots, f_{n}$ из $\mathscr{H}(D)$ такие, что $F(z, t) \equiv f_{k}(z)$ при $t \in\left(t_{k-1}, t_{k}\right]$, $k=1, \ldots, n$. В [47] (см. также [44], [41]) установлен следующий результат.

Теорема 13. Пусть $\mathscr{K}$ - компактное подмножество пространства $\mathscr{H}(D)$ u $\mathscr{Q}=\operatorname{ext} \overline{\mathrm{co}} \mathscr{K}-$ совокупность крайних точек его замкнутой выпуклой оболочки. Тогда для любой $\mathscr{K}$-значной функции $F(z, t)$ из $\mathfrak{F}(D, I)$ найдется последовательность ступенчатых $\mathscr{Q}$-значных функиий $F_{n}(z, t), n=1,2, \ldots$, такая, что $\left\{F_{n}\right\}$ слабо сходится в $\mathfrak{F}(D, I)$ к функиии $F$.

Результаты о слабой сходимости инфинитезимальных производящих функций естественным образом приводят к инфинитезимальному описанию полугруппы $\mathfrak{L}$ и позволяют лучше понять ее структуру. Как уже отмечалось выше, Лёвнер показал, что каждую функцию $f$ из $\mathfrak{L}$ можно аппроксимировать в топологии локально равномерной сходимости отображениями $w_{T, 0}^{P}$ с некоторым $T>0$ и инфинитезимальной производящей функцией $P(z, t)$ вида (13). Чтобы придать этому результату несколько иное звучание, введем в рассмотрение класс $\mathscr{C}$ голоморфных в единичном круге $\mathbb{D}$ функций $p$, удовлетворяющих условиям $\operatorname{Re} p(z)>0$ при $z \in \mathbb{D}$ и $p(0)=1$. В литературе этот класс функций часто называют классом Каратеодори. Заметим, что $\mathscr{C}$ является выпуклым компактным подмножеством пространства $\mathscr{H}(\mathbb{D})$ и множество его крайних точек имеет вид

$$
\operatorname{ext} \mathscr{C}=\left\{p(z)=\frac{1+\varkappa z}{1-\varkappa z}: \varkappa \in \mathbb{T}\right\} .
$$

В частности, формулу Рисса-Герглотца можно интерпретировать с точки зрения выпуклого анализа как реализацию теорем Крейна-Мильмана и Шоке (см., 
например, [48]). Таким образом, отображения $w_{T, 0}^{P}, T>0$, с ext $\mathscr{C}$-значными инфинитезимальными производящими функциями $P(z, t)$ образуют плотное в $\mathfrak{L}$ подмножество относительно локально равномерной в $\mathbb{D}$ сходимости. Заметим также, что в случае, когда $P(\cdot, t) \in \mathscr{C}$ для почти всех $t$, выполняется равенство $\left(w_{t, 0}^{P}\right)^{\prime}(0)=e^{-t}$, которое непосредственно выводится из уравнения (11). Кроме того, для любого $\beta \in(0,1)$ множество

$$
\left\{f \in \mathfrak{L}: f^{\prime}(0)=\beta\right\}
$$

является компактным относительно топологии локально равномерной в $\mathbb{D}$ сходимости. Далее, из теоремы 12 следует, что совокупность $\mathscr{C}$-значных функций в пространстве $\mathfrak{F}(\mathbb{D},[0, T])$ образует компактное относительно слабой сходимости множество. Это и теорема 11 показывают, что при фиксированном $T>0$ множество отображений $w_{T, 0}^{P}$ с $\mathscr{C}$-значными инфинитезимальными производящими функциями $P(z, t)$ также образует компактное подмножество в $\mathfrak{L}$. Резюмируя изложенное выше, приходим к следующему результату.

Теорема 14. Для того чтобы голоморфная в $\mathbb{D}$ функиия $f$ c $f(0)=0$ u $f^{\prime}(0)=\beta>0$ принадлежала полугруппе $\mathfrak{L}$, необходимо и достаточно, чтобы она допускала представление в виде

$$
f(z)=w_{T, 0}^{P}(z)
$$

где $T=-\ln \beta$, а функиия $P(z, t)$ голоморфна по $z$ в $\mathbb{D}$, измерима по $t$ на $[0, T]$ u такова, что $P(\cdot, t) \in \mathscr{C}$ для почти всех $t \in[0, T]$.

Как видно из предыдущего, конус инфинитезимальных преобразований полугруппы $\mathfrak{L}$ описывается формулой $v(z)=-\alpha z p(z)$, где $\alpha \geqslant 0$, а $p \in \mathscr{C}$. С точки зрения инфинитезимального описания полугруппы наиболее естественный способ выделения подполугрупп состоит в сужении конуса инфинитезимальных преобразований. В связи с этим введем ряд обозначений и определений.

Пусть $\mathscr{K}$ - некоторое подмножество класса Каратеодори $\mathscr{C}$. Через $\mathfrak{L}(\mathscr{K})$ обозначим совокупность отображений $f$ из $\mathfrak{L}$ вида $f(z)=w_{T, 0}^{P}(z)$, где $T \geqslant 0$, а инфинитезимальная производящая функция $P(z, t)$ удовлетворяет условию $P(\cdot, t) \in \mathscr{K}$ для почти всех $t \in[0, T]$. В силу теоремы 14 имеет место равенство $\mathfrak{L}(\mathscr{C})=\mathfrak{L}$. Приведем два результата, касающиеся структуры полугруппы $\mathfrak{L}(\mathscr{K})$.

TеОрема 15 (см. [47], [41]). Пусть $\mathscr{K}$ - замкнутое выпуклое собственное подмножество класса $\mathscr{C}$. Тогда $\mathfrak{L}(\mathscr{K})$ представляет собой замкнутую собственную подполугруппу полугруппы $\mathfrak{L}$.

Под замкнутостью подполугруппы $\mathfrak{L}(\mathscr{K})$ понимается следующее свойство: если последовательность функций $f_{n}, n=1,2, \ldots$, из $\mathfrak{L}(\mathscr{K})$ сходится локально равномерно в $\mathbb{D}$ к некоторой функции $f$ из $\mathfrak{L}$, то предельная функция $f$ также принадлежит подполугруппе $\mathfrak{L}(\mathscr{K})$.

Пусть теперь $h$ - произвольная функция класса $\mathscr{C}$. Если положить $P(z, t) \equiv$ $h(z)$, то семейство отображений

$$
\phi_{t}^{h}(z)=w_{t, 0}^{P}(z)
$$

$t \geqslant 0$, можно рассматривать как однопараметрическую полугруппу $t \mapsto \phi_{t}^{h}$ в $\mathfrak{L}$ с инфинитезимальной образующей $v(z)=-z h(z)$. В этих обозначениях сформулируем следующий результат. 
ТеОрема 16 (см. [47], [41]). Пусть $\mathscr{K}$ - замкнутое выпуклое подмножество класса $\mathscr{C}$ и $\mathscr{Q}=\operatorname{ext} \mathscr{K}-$ совокупность его крайних точек. Тогда функции вида

$$
\phi(z)=\phi_{\tau}^{h_{1}} \circ \cdots \circ \phi_{\tau}^{h_{n}}(z),
$$

где $\tau \geqslant 0, h_{k} \in \mathscr{Q}(k=1, \ldots, n ; n=1,2, \ldots)$, образуют плотное в $\mathfrak{L}(\mathscr{K})$ подмножество относительно топологии локально равномерной в $\mathbb{D}$ сходимости.

В случае $\mathscr{K}=\mathscr{C}$ подполугруппа $\mathfrak{L}(\mathscr{K})$ совпадает со всей полугруппой $\mathfrak{L}$. Множество $\mathscr{Q}$ в этом случае составляют функции

$$
g_{\varkappa}(z)=\frac{1+\varkappa z}{1-\varkappa z}
$$

$\varkappa \in \mathbb{T}$. При этом

$$
\phi_{\tau}^{g_{\varkappa}}(z)=K_{\varkappa}^{-1}\left(e^{-\tau} K_{\varkappa}(z)\right)
$$

где

$$
K_{\varkappa}(z)=\frac{z}{(1+\varkappa z)^{2}}
$$

- функция Кёбе. Отсюда видно, что $\phi_{\tau}^{g_{\varkappa}}$ отображает $\mathbb{D}$ на область, которая получается из единичного круга проведением радиального разреза, выходящего из точки $\bar{\varkappa}$ единичной окружности. Длина разреза зависит от величины $\tau$. В этом частном случае результат теоремы 16 совпадает с леммой из [5], которую де Бранж использовал в первоначальной версии доказательства гипотезы Бибербаха.

В работах [44], [49] были изучены эволюционные уравнения и вопрос вложимости в эволюционные семейства для полугрупп конформных отображений полосы и полуплоскости. Отметим также, что эволюционные семейства конформных отображений и уравнение Лёвнера-Куфарева широко используются в различных приложениях (см., например, [50], [51]).

\section{5. Параметрический метод теории однолистных функций}

Под однолистной функцией в области $D \subset \mathbb{C}$ понимается аналитическая функция $f$, отображающая эту область взаимно однозначно на другую область комплексной плоскости, т. е. $f\left(z_{1}\right) \neq f\left(z_{2}\right)$ при $z_{1} \neq z_{2}, z_{1}, z_{2} \in D$. Эти функции играют важную роль как с точки зрения теории конформного отображения, так и с точки зрения приложений. В частности, часто в приложениях однолистность связывается с физической реализуемостью математической модели. Центральную роль в теории однолистных функций играют экстремальные задачи. Сама проблема конформного отображения может быть рассмотрена как экстремальная задача. Кроме того, к оценкам функционалов и отысканию областей значений систем функционалов, зависящих от значений однолистной функции и ее производных в фиксированной точке, сводятся многие задачи, связанные с геометрией конформного отображения. Ряд результатов этого направления в связи с их геометрическим смыслом получили названия теорем искажения, вращения, роста и т. д. Следует отметить также, что экстремальные задачи теории однолистных функций не укладываются в рамки классического вариационного исчисления и их исследование на протяжении нескольких десятилетий стимулировало возникновение и совершенствование ряда тонких 
специальных методов. Мы не будем останавливаться подробно на описании всех методов и результатов теории однолистных функций. Этому посвящено много обзоров (см., например, [52]-[54]). Отметим лишь некоторые особенности ряда методов.

Одним из основных объектов изучения теории однолистных функций является класс $\mathscr{S}$ голоморфных и однолистных в единичном круге $\mathbb{D}$ функций $f$, нормированных условиями $f(0)=0, f^{\prime}(0)=1$. Существенная нелинейность этого объекта создает основные трудности построения на нем эффективного вариационного исчисления. Первые результаты теории однолистных функций были получены методом площадей, который восходит к работам Гронуолла, Бибербаха, Фабера и основывается на простом геометрическом принципе неотрицательности площади, выражаемой, как правило, в терминах коэффициентов разложения самой функции или связанного с ней аналитического выражения по какой-либо ортонормированной системе. Достаточно полное представление об этом методе и его приложениях можно получить из монографий [55], [56]. Именно методом площадей была впервые получена точная оценка второго коэффициента тейлоровского разложения функции класса $\mathscr{S}$, что побудило Бибербаха сформулировать в 1916 г. гипотезу о справедливости неравенства $\left|c_{n}\right| \leqslant n$ для любой функции $f(z)=z+c_{2} z^{2}+\cdots$ класса $\mathscr{S}$. Эта гипотеза на долгое время определила направление развития теории однолистных функций. Первое продвижение в ее решении было сделано лишь в 1923 г. Лёвнером [3], который подтвердил гипотезу для $n=3$ и заложил основы нового метода, ставшего впоследствии известным как параметрический метод. Именно этим методом де Бранж [5], [6] получил полное решение гипотезы Бибербаха в 1984 г.

Идея вариационного метода ближе к классическому вариационному исчислению и связана с описанием локальной структуры классов однолистных функций в пространстве всех голоморфных функций. Класс $\mathscr{S}$ представляет собой компактное подмножество линейного пространства $\mathscr{H}(\mathbb{D})$ с метризуемой топологией. Вариация функции $f \in \mathscr{S}$ (или вариационная формула) заключается в выделении в $\mathscr{H}(\mathbb{D})$ таких направлений, сдвиг вдоль которых из $f$ приводит к функциям, расстояние от которых до класса $\mathscr{S}$ представляет собой величину более высокого порядка малости, чем длина сдвига. Способом выделения таких направлений различаются известные методы: вариационно-геометрический Лаврентьева [57], граничных вариаций Шиффера [58], внутренних вариаций Шиффера-Голузина [52]. Вариация функций класса $\mathscr{S}$ приводит к вариации дифференцируемых по Гато функционалов и к необходимым условиям локального экстремума, которые выражаются в виде дифференциального уравнения для экстремальных функций. Исследование, например, уравнения Шиффера-Голузина, получаемого методом внутренних вариаций, приводит к качественной информации об экстремалях. Доведению же решения задачи до конца часто мешает сложность получаемого уравнения и присутствие в нем неизвестных параметров. Кроме того, с точки зрения общего вариационного исчисления уравнение Шиффера-Голузина представляет собой лишь необходимое условие экстремума и не разделяет даже локальных максимумов и минимумов. В классическом вариационном исчислении вопрос о разделении локальных максимумов и минимумов решается посредством второй вариации. В задачах теории 
однолистных функций ситуация существенно усложняется бесконечномерностью пространства $\mathscr{H}(\mathbb{D})$ и трудностями, связанными с изучением структуры класса $\mathscr{S}$ как подмножества этого пространства. Тем не менее плодотворность рассмотрения второй вариации была продемонстрирована в [59].

Метод экстремальных метрик связан с изучением дифференциально-геометрических особенностей экстремальных конформных отображений. В работах Грётча и Тейхмюллера этот метод основывался на неравенствах, связывающих длину и площадь. Важную роль в его развитии сыграло введенное Альфорсом и Бёрлингом понятие экстремальной длины (или модуля) семейства кривых. Применение этого метода основывается на установлении связи исследуемой задачи теории однолистных функций с некоторой экстремально-метрической проблемой. Экстремальная метрика при этом появляется как решение соответствующей экстремально-метрической проблемы и порождается некоторым квадратичным дифференциалом. Именно через квадратичный дифференциал пролегает связь между методом экстремальных метрик и вариационным методом, которые в ряде исследований взаимно дополняют друг друга. Представление о методе экстремальных метрик можно получить по монографии [60] и обзору [54].

Геометрический аспект метода экстремальных метрик позволяет сочетать его также с методом симметризации. Идея симметризации возникла при исследовании задач геометрии и математической физики. Благодаря геометрическим и физическим интерпретациям аналитических функций и конформного отображения, симметризация получила приложения в теории однолистных функций (см., например, [60]-[63]). Современное состояние метода симметризации и смежных вопросов можно найти в недавней монографии [64].

Во многом трудности построения эффективного вариационного исчисления на классах однолистных функций обусловлены существенной нелинейностью объекта. Сумма двух однолистных функций не обязательно будет однолистной. С другой стороны, операция композиции (если она определена) не нарушает однолистности. Именно на этот факт обращает внимание Лёвнер при выделении полугруппы L. Как уже отмечалось выше, вопрос инфинитезимального описания полугруппы $\mathfrak{L}$ Лёвнер решил аппроксимативно. При этом он показал, что предел

$$
f(z)=\lim _{t \rightarrow \infty} e^{t} w_{t, 0}^{P}(z)
$$

существует и представляет собой функцию $f$ из класса $\mathscr{S}$ для любой инфинитезимальной производящей функции $P(z, t)$ вида $(13)$ на $[0, \infty)$. Кроме того, полученные таким образом функции $f$ образуют плотное в $\mathscr{S}$ подмножество $\mathscr{S}_{L}$. Следовательно, точные оценки непрерывных относительно топологии локально равномерной в $\mathbb{D}$ сходимости функционалов на классах $\mathscr{S}$ и $\mathscr{S}_{L}$ совпадают. Это обстоятельство лежит в основе классического варианта параметрического метода. Выразив тейлоровские коэффициенты функции $f$ класса $\mathscr{S}_{L}$ в терминах унимодулярной функции $\varkappa(t)$ из $(13)$, Лёвнер впервые подтвердил гипотезу Бибербаха в случае $n=3$.

Новые возможности параметрического метода открыла работа Голузина [65], в которой он нашел неожиданное его применение к проблеме вращения. Эта проблема также возникла из работы Бибербаха [66], в которой он установил 
для функций $f \in \mathscr{S}$ оценку

$$
\left|\arg f^{\prime}\left(z_{0}\right)\right| \leqslant 2 \ln \frac{1+\left|z_{0}\right|}{1-\left|z_{0}\right|},
$$

где рассматривается ветвь аргумента, обращающаяся в нуль при $z_{0}=0$. Не нарушая общности, можно считать, что $z_{0}=r, 0<r<1$. Геометрически величина $\arg f^{\prime}(r)$ выражает угол, на который поворачивается касательная при движении точки вдоль образа отрезка $[0, r]$ при отображении его функцией $f$. Неравенство, полученное Бибербахом, оказалось недостижимым в классе $\mathscr{S}$. Вопрос о точных неравенствах для $\arg f^{\prime}(r)$ и составил проблему вращения. Впервые используя для таких задач параметрический метод, Голузин получил следующие оценки:

$$
\arg f^{\prime}(r) \leqslant\left\{\begin{array}{lll}
4 \arcsin r & \text { при } & 0<r \leqslant \frac{1}{\sqrt{2}}, \\
\pi+\ln \frac{r^{2}}{1-r^{2}} & \text { при } & \frac{1}{\sqrt{2}}<r<1 .
\end{array}\right.
$$

Точность неравенства при $r \leqslant 1 / \sqrt{2}$ была установлена предъявлением экстремальной функции. Вопрос о точности неравенств при $r>1 / \sqrt{2}$ впервые решил Базилевич [67]. Позже Голузин использовал параметрический метод, чтобы единообразно вывести основные результаты теории однолистных функций. Различные аспекты этого метода и его применения отражены во многих работах (см., например, [19], [35], [52], [63], [68], [69]). При этом часто отмечалось, что параметрический метод хорош в получении точных оценок, но не дает информации о единственности экстремалей и их описания.

Трудности, возникающие при решении вопроса единственности экстремалей в рамках параметрического метода, обусловлены были прежде всего тем, что экстремальная задача рассматривалась на классе $\mathscr{S}_{L}$. Из результатов работ [34], [42] следовало, что замена в формуле (14) инфинитезимальной производящей функции $P(z, t)$ специального вида (13) на произвольную измеримую $\mathscr{C}$-значную функцию приводит к параметрическому представлению всего класса $\mathscr{S}$. Этот результат можно сформулировать в следующем виде (см., например, [35]).

Теорема 17. Для того чтобы голоморфная в $\mathbb{D}$ функиия $f$ принадлежала классу $\mathscr{S}$, необходимо и достаточно, чтобы ее можно было представить в виде (14) с некоторой функиией $P(z, t)$, голоморфной по $z$ в $\mathbb{D}$, измеримой nо t на $[0, \infty)$ и такой, что $P(\cdot, t) \in \mathscr{C}$ для почти всех $t \in[0, \infty)$.

Как отмечалось в предыдущем разделе, сужение конуса инфинитезимальных преобразований полугруппы $\mathfrak{L}$ приводит к подполугруппам $\mathfrak{L}(\mathscr{K})$. Аналогично, сужение множества инфинитезимальных производящих функций $P(z, t)$ в формуле (14) приводит к выделению подклассов однолистных функций. Более точно, пусть $\mathscr{K}$ - некоторое подмножество класса Каратеодори $\mathscr{C}$. Через $\mathscr{S}(\mathscr{K})$ будем обозначать класс функций $f$, которые представимы формулой $(14)$ с некоторой функцией $P(z, t)$, голоморфной по $z$ в $\mathbb{D}$, измеримой по $t$ на $[0, \infty)$ и такой, что $P(\cdot, t) \in \mathscr{K}$ для почти всех $t \in[0, \infty)$. Конкретизация подмножества $\mathscr{K} \subset \mathscr{C}$ приводит к различным известным классам однолистных функций. В работах [47], [41] изучалась структура этих классов. В частности, оказалось, что замыкание классов $\mathscr{S}(\mathscr{K})$ описывается такой же структурой. 
Теорема 18. Пусть $\mathscr{K}$ - некоторое подмножество класса $\mathscr{C}$. Тогда замъкание $\overline{\mathscr{S}(\mathscr{K})}$ в топологии локально равномерной в $\mathbb{D}$ сходимости класса $\mathscr{S}(\mathscr{K})$ совпадает с классом $\mathscr{S}(\overline{\mathrm{co}} \mathscr{K})$, m.e.

$$
\overline{\mathscr{S}(\mathscr{K})}=\mathscr{S}(\overline{\mathrm{co}} \mathscr{K})
$$

где со $\mathscr{K}$ - замкнутая выпуклая оболочка множества $\mathscr{K}$.

Первым препятствием в анализе вопроса единственности экстремалей, как отмечалось выше, было то, что классический вариант параметрического метода базировался на параметрическом представлении лишь всюду плотного подмножества $\mathscr{S}_{L}$ класса $\mathscr{S}$. Параметрическое представление класса всех однолистных функций позволило решить вопрос единственности экстремалей в некоторых частных случаях. Однако во многих задачах теории однолистных функций возникают трудности другой природы. Это связано с тем, что одна и та же функция $f$ класса $\mathscr{S}$ может быть получена по формуле (14) с существенно различными инфинитезимальными производящими функциями $P(z, t)$. Преодоление этих трудностей оказалось возможным с использованием полугрупповой структуры отображений $w_{t, s}^{P}[70]$. Развитие этого подхода позволило также получить теорему единственности граничных функций системы

$$
I(f)=\left(I_{1}(f), \ldots, I_{4}(f)\right)
$$

вещественнозначных функционалов, определяемых на классе $\mathscr{S}$ формулами

$$
I_{1}(f)+i I_{2}(f)=\ln \frac{f\left(z_{0}\right)}{z_{0}}, \quad I_{3}(f)+i I_{4}(f)=\ln \frac{z_{0} f^{\prime}\left(z_{0}\right)}{f\left(z_{0}\right)},
$$

где $z_{0}$ - фиксированная точка единичного круга $\mathbb{D}$, а под логарифмами понимаются непрерывные ветви, обращающиеся в нуль при $z_{0}=0$.

Многие внутренние задачи теории однолистных функций включаются в исследование областей значений систем функционалов, зависящих от значений однолистной функции и ее производной [71]. Широкий круг задач, включающий теоремы искажения и вращения, сводится к изучению множества

$$
D\left(z_{0}\right)=\{I(f): f \in \mathscr{S}\} .
$$

В частности, теорема вращения состоит в точной оценке суммы $I_{2}(f)+I_{4}(f)$.

В силу компактности класса $\mathscr{S}$ и непрерывности функционалов $I_{1}, \ldots, I_{4}$ относительно топологии локально равномерной в $\mathbb{D}$ сходимости, $D\left(z_{0}\right)$ представляет собой ограниченное замкнутое подмножество в $\mathbb{R}^{4}$. Функция $f$ из класса $\mathscr{S}$ называется граничной относительно системы $I$, если точка $I(f)$ принадлежит границе множества $D\left(z_{0}\right)$. Информация о границе множества $D\left(z_{0}\right)$ позволяет получать оценки конкретных функционалов, зависящих от значений функции и ее производной в классе $\mathscr{S}$, а экстремалями этих функционалов являются граничные функции. Впервые описание границы множества $D\left(z_{0}\right)$ в замкнутой форме было дано в [72]. Позже в [42] была получена параметризация системы $I$, которая позволила доказать выпуклость области $D\left(z_{0}\right)$ и получить более простое ее описание. В работах [73], [74] установлена единственность граничных функций и дано их описание. В частности, имеет место следующий результат.

Теорема 19. Пусть $z_{0} \in \mathbb{D}, z_{0} \neq 0$. Тогда $D\left(z_{0}\right)$ замкнутое выпуклое ограниченное множество в $\mathbb{R}^{4}$, каждую граничную точку которого вносит единственная функиия класса $\mathscr{S}$. 
Полная формулировка этого результата (см. [73], [74]) содержит также описание вида граничных функций. Наличие на границе множества $D\left(z_{0}\right)$ прямолинейных отрезков является причиной неединственности экстремалей в конкретных задачах об оценках функционалов. В частности, этим объясняется неединственность экстремалей в теореме вращения при $1 / \sqrt{2}<\left|z_{0}\right|<1$. В этом случае экстремальные функции образуют однопараметрическое семейство. Каждая функция этого семейства вносит точку отрезка на границе множества $D\left(z_{0}\right)$. Полугрупповые свойства экстремальных эволюционных семейств позволили выяснить структуру экстремалей и получить их полное описание.

\section{6. Полугруппы вероятностных производящих функций и ветвящиеся процессы}

Целью данного раздела является попытка показать связи между динамикой голоморфного отображения и некоторыми вопросами теории ветвящихся случайных процессов. Мы будем придерживаться терминологии хорошо известных монографий [75]-[77], из которых можно также почерпнуть необходимые сведения о ветвящихся процессах.

6.1. Задача вложения. Ветвящиеся процессы, как правило, описывают развитие популяции однотипных частиц, которые независимо друг от друга воспроизводят другие частицы того же вида. Под частицами могут пониматься животные, бактерии, нейтроны в цепных реакциях и т. д. Простейшей моделью ветвящегося процесса с дискретным временем является так называемый процесс Гальтона-Ватсона. Предполагается, что в начальный момент времени популяцию составляет одна частица, которая в следующем поколении превращается в $k$ частиц с вероятностью $p_{k} \geqslant 0, k=0,1, \ldots, \sum_{k=0}^{\infty} p_{k}=1$. Частицы первого поколения независимо друг от друга с тем же вероятностным законом каждая формируют второе поколение и т. д. Обозначим через $\xi_{0}=1, \xi_{1}, \xi_{2}, \ldots$ число частиц в нулевом, первом, втором, ... поколениях соответственно. Тогда $\xi_{0}, \xi_{1}, \xi_{2}, \ldots$ образуют однородную цепь Маркова. Начальными данными этого процесса считается распределение вероятностей $\mathrm{P}\left(\xi_{1}=k\right)=p_{k}, k=0,1,2, \ldots$. Важным инструментом в теории ветвящихся процессов являются вероятностные производящие функции. В частности, процесс Гальтона-Ватсона полностью определяется вероятностной производящей функцией случайной величины $\xi_{1}$ :

$$
f(z)=\mathrm{E} z^{\xi_{1}}=\sum_{k=0}^{\infty} p_{k} z^{k}
$$

Предположения, сделанные относительно процесса, приводят к тому, что производящей функцией случайной величины $\xi_{n}$, выражающей объем $n$-го поколения, оказывается $n$-я итерация функции $f$, т. е.

$$
\mathrm{E} z^{\xi_{n}}=f^{n}(z)=f \circ \cdots \circ f(z) .
$$

Таким образом, вероятностную производящую функцию $f$ можно рассматривать как начальные данные процесса, а динамика процесса описывается натуральными итерациями функции $f$. 
Вопрос о возможности вложения процесса Гальтона-Ватсона с производящей функцией $f$ в однородный марковский ветвящийся процесс с непрерывным временем эквивалентен (см. [75]) вопросу существования дробных итераций функции $f$, или ее вложения в однопараметрическую полугруппу $t \mapsto f^{t}$. При этом требуется, чтобы все итерации $f^{t}, t \geqslant 0$, также были вероятностными производящими функциями. Введем некоторые определения и сформулируем эту задачу в несколько уточненном виде.

Через $\mathfrak{P}^{+}$обозначим совокупность всех вероятностных производящих функций $f$, т. е. таких, которые представимы в виде суммы степенного ряда: $f(z)=$ $\sum_{k=0}^{\infty} p_{k} z^{k}$, где $p_{k} \geqslant 0$ и $\sum_{k=0}^{\infty} p_{k}=1$. Очевидно, что вероятностная производящая функция $f$ является голоморфной в единичном круге $\mathbb{D}$ и непрерывной в его замыкании $\overline{\mathbb{D}}$. Кроме того, $|f(z)| \leqslant 1$ при $z \in \mathbb{D}$ и если $f$ и $g$ - две вероятностные производящие функции, то их композиция $h=f \circ g$ также является вероятностной производящей функцией. Таким образом, $\mathfrak{P}^{+}$представляет собой полугруппу относительно операции композиции. Если из $\mathfrak{P}^{+}$исключить функцию $f(z) \equiv 1$, которая отвечает вырожденной случайной величине, принимающей значение нуль с вероятностью 1 , то $\mathfrak{P}^{+}$будет собственной подполугруппой полугруппы $\mathfrak{P}$. Как и в работе [78], концепцию вложимости уточним посредством следующего определения.

ОПРЕДЕЛЕНИЕ 3. Вероятностная производящая функция $f$ будет называться вложимой, если найдется однопараметрическая полугруппа $t \mapsto f^{t}$ в $\mathfrak{P}^{+}$такая, что $f^{1}=f$. Класс всех вложимых вероятностных производящих функций обозначим через $\mathscr{E}\left(\mathfrak{P}^{+}\right)$.

Другими словами, вероятностная производящая функция $f$ принадлежит классу $\mathscr{E}\left(\mathfrak{P}^{+}\right)$в том и только том случае, если она определяет процесс Гальтона-Ватсона, допускающий вложение в однородный марковский ветвящийся процесс с непрерывным временем. Впервые класс вложимых вероятностных производящих функций был рассмотрен в [79], где была поставлена задача его описания. В этой работе было доказано, что если $f(0)=0$ и $f$ - целая функция, то она не будет вложимой, а также установлен следующий результат.

Теорема 20. Пусть $f(z)=\sum_{k=0}^{\infty} p_{k} z^{k}$ - вероятностная производящая функиия и $p_{0}=0,0<p_{1}<1$. Тогда $f \in \mathscr{E}\left(\mathfrak{P}^{+}\right)$в том и только том случае, если неотрицательны числа $b_{n}, n=2,3, \ldots$, определяемые из рекуррентных соотношений

$$
b_{n}=\frac{1}{p_{1}-p_{1}^{n}} \sum_{j=1}^{n-1} b_{j}\left[\beta_{n j}-(n-j+1) p_{n-j+1}\right],
$$

где $b_{1}=\ln p_{1}$, a $\beta_{n j}-$ коэфбициент при $z^{n}$ в разложении $[f(z)]^{j}$ в степенной ряд.

Дальнейшее продвижение в решении проблемы вложения было получено в работе [78]. 
Tеорема 21. Пусть $f \in \mathfrak{P}^{+}$голоморфна в точке $z=1 u f^{\prime}(1)>1, f(0)=0$, $f^{\prime}(0)>0$. Допустим также, что $f$ голоморфна в $\mathbb{C} \backslash S$, где $S$ - счетное замкнутое подмножество в расширенной комплексной плоскости. Тогда $f \in \mathscr{E}\left(\mathfrak{P}^{+}\right)$в том и только том случае, если $f$ является дробно-линейной функиией.

Таким образом, если вероятностная производящая функция $f$ мероморфна в $\mathbb{C}$, то она не будет вложимой, за исключением случая дробно-линейной функции. Кроме того, в [78] были получены следующие необходимые условия вложимости (см. также [77]).

Теорема 22. Пусть $f \in \mathfrak{P}^{+} u q$ - наименъший неотрицательный корень уравнения $f(x)=x$. Допустим также, что $f^{(\mathrm{iv})}(q)<\infty$ (условие содержательно лишь в случае $q=1)$. Тогда имент место следующие утверждения:

(a) если $3\left(f^{\prime \prime}(q)\right)^{2}-2 f^{\prime}(q) f^{\prime \prime \prime}(q)>0$, mo $f \notin \mathscr{E}\left(\mathfrak{P}^{+}\right)$;

(b) если $3\left(f^{\prime \prime}(q)\right)^{2}-2 f^{\prime}(q) f^{\prime \prime \prime}(q)=0, q>0$, u $f \in \mathscr{E}\left(\mathfrak{P}^{+}\right)$, то $f$ является дробно-линейной функиией.

Вопрос описания класса $\mathscr{E}\left(\mathfrak{P}^{+}\right)$поднимался в монографиях [75], [77] и многих других работах в связи с тем, что аналитический аппарат изучения однородных марковских ветвящихся процессов с непрерывным временем значительно шире, чем средства изучения процессов Гальтона-Ватсона. В работах [80], [81] были получены критерии и простые необходимые условия вложимости. В частности, имеет место следующий общий критерий вложимости [81].

Tеорема 23. Пустъ $f \in \mathfrak{P}^{+} u f(z) \not \equiv 1$. Тогда $f \in \mathscr{E}\left(\mathfrak{P}^{+}\right)$в том и только том случае, если существует решение $v$ уравнения

$$
v \circ f(z)=v(z) f^{\prime}(z),
$$

которое представляет собой голоморфную в $\mathbb{D}$ функиию, удовлетворяющую условиям $v^{\prime}(0)=-1$ и $v^{(n)}(0) \geqslant 0$ при $n=2,3, \ldots$.

Прежде чем приступить к формулировке других результатов из названных выше работ, сделаем некоторые замечания и введем необходимые обозначения.

Пусть $f$ - вероятностная производящая функция. Наименьший неотрицательный корень $q$ уравнения $f(x)=x$ имеет важный как вероятностный, так и динамический смысл. Прежде всего, $q$ является вероятностью вырождения процесса Гальтона-Ватсона с производящей функцией $f$. С точки зрения динамики голоморфного отображения $q$ является точкой Данжуа-Вольфа функции $f$, т. е. $f^{n}(z) \rightarrow q$ локально равномерно в $\mathbb{D}$ при $n \rightarrow \infty$. Заметим также, что для любой вероятностной производящей функции $f$ точка Данжуа-Вольфа $q$ принадлежит отрезку $[0,1]$, поскольку $0 \leqslant f^{n}(0)<1$ при всех $n=1,2, \ldots$ и $q=\lim _{n \rightarrow \infty} f^{n}(0)$.

Через $\mathfrak{P}^{+}[q], 0 \leqslant q \leqslant 1$, обозначим совокупность функций $f$ из $\mathfrak{P}^{+}$, для которых $q$ является точкой Данжуа-Вольфа, т. е. соответствующий процесс Гальтона-Ватсона имеет вероятность вырождения $q$. Очевидно, что $\mathfrak{P}^{+}[q]$ является подполугруппой полугруппы $\mathfrak{P}^{+}$.

Другой способ классификации процессов Гальтона-Ватсона связан с математическим ожиданием числа потомков от одной частицы в следующем поколении. Эта величина выражается в терминах производящей функции $f$ как угловая производная $f^{\prime}(1)$. Если $f^{\prime}(1)<1$, то соответствующий процесс называется 
докритическим; совокупность функций $f$ из $\mathfrak{P}^{+}$, удовлетворяющих этому условию, будем обозначать $\mathfrak{P}_{\text {sub }}^{+}$. Аналогично выделяется множество $\mathfrak{P}_{\mathrm{c}}^{+}$функций $f$ из $\mathfrak{P}^{+}$, которые удовлетворяют условию $f^{\prime}(1)=1$ и соответствуют критическим процессам. Через $\mathfrak{P}_{\text {sup }}^{+}$обозначим совокупность вероятностных производящих функций $f$, удовлетворяющих условию $f^{\prime}(1)>1$ (или $f^{\prime}(1)=+\infty$ ) и соответствующих надкритическим процессам. Выделенные подмножества также являются подполугруппами полугруппы $\mathfrak{P}^{+}$. Кроме того, выполняются следующие соотношения:

$$
\mathfrak{P}^{+}[1]=\mathfrak{P}_{\mathrm{c}}^{+} \cup \mathfrak{P}_{\text {sub }}^{+}, \quad \mathfrak{P}_{\text {sup }}^{+}=\bigcup_{0 \leqslant q<1} \mathfrak{P}^{+}[q]
$$

Будем считать, что единичный элемент, т. е. тождественное преобразование $f(z) \equiv z$, принадлежит всем выделенным подполугруппам. Кроме того, их структура как выпуклых подмножеств в пространстве $\mathscr{H}(\mathbb{D})$ отражена в следующих двух результатах [80].

Теорема 24. Голоморфная в $\mathbb{D}$ функиия $f$ принадлежит $\mathfrak{P}_{\mathrm{c}}^{+}$(соответственно $\mathfrak{P}_{\mathrm{sub}}^{+}$) в том и только том случае, если она представима в виде

$$
f(z)=1+\sum_{k=1}^{\infty} \frac{\lambda_{k}}{k}\left(z^{k}-1\right)
$$

где $\lambda_{k} \geqslant 0 u \sum_{k=1}^{\infty} \lambda_{k}=1$ (соответственно $\left.\sum_{k=1}^{\infty} \lambda_{k}<1\right)$.

Теорема 25. Голоморфная в $\mathbb{D}$ функиия $f$ принадлежит $\mathfrak{P}^{+}[q], 0 \leqslant q<1$, в том и только том случае, если она представима в виде

$$
f(z)=q+(1-q) \sum_{k=1}^{\infty} \lambda_{k} \frac{z^{k}-q^{k}}{1-q^{k}}
$$

где $\lambda_{k} \geqslant 0 u \sum_{k=1}^{\infty} \lambda_{k}=1$.

Если процесс докритический, то вероятность вырождения $q$ равна 1 , а в случае надкритического процесса $q \in[0,1)$. Для этих процессов условие вложимости имеет следующий вид [80].

Теорема 26. Некритический прочесс Гальтона-Ватсона с вероятностью вырождения q и производящей функиией $f$ допускает вложение в однородный марковский ветвящийся процесс с непрерывным временем в том и только том случае, если $f^{\prime}(z) \neq 0$ при $z \in \mathbb{D}$ и существует локально равномерный в $\mathbb{D}$ предел

$$
\lim _{n \rightarrow \infty} \frac{q-f^{n}(z)}{\left(f^{n}\right)^{\prime}(z)}=u(z),
$$

удовлетворяющий условиям $u^{(k)}(0) \geqslant 0$ при $k=2,3, \ldots$.

В критическом случае имеет место следующий результат [80]. 
Теорема 27. Критический процесс Гальтона-Ватсона с производящей функиией $f$ допускает вложение в однородньй марковский ветвящийся процесс с непрерывным временем в том и только том случае, если $f^{\prime}(z) \neq 0$ при $z \in \mathbb{D}$ и существует локально равномерный в $\mathbb{D}$ предел

$$
\lim _{n \rightarrow \infty} \frac{\left(f^{n}\right)^{\prime}(0)}{\left(f^{n}\right)^{\prime}(z)}=u(z)
$$

удовлетворяющий условиям $u(f(0))=f^{\prime}(0) u u^{(k)}(0) \geqslant 0$ nри $k=2,3, \ldots$.

Приведем теперь ряд необходимых условий вложимости. Следующее утверждение касается вероятностных производящих функций, отвечающих надкритическим процессам. При этом, когда вероятность вырождения равна нулю, результат формулируется в терминах начальных вероятностей. В случае $0<q<1$ необходимое условие вложимости формулируется, как и в соответствующем результате Карлина и Макгрегора, в терминах производных вероятностной производящей функции в точке $z=q$. Отличие состоит в том, что удается понизить максимальный порядок входящих производных с третьего до второго [80].

Tеорема 28. Пусть $f \in \mathfrak{P}^{+}[q], 0 \leqslant q<1, u f(z) \not \equiv z$. Тогда имеют место следующие утверждения:

(a) если

$$
\frac{f^{\prime \prime}(q)}{f^{\prime}(q)\left(1-f^{\prime}(q)\right)}>\frac{2}{1-q}
$$

mo $f \notin \mathscr{E}\left(\mathfrak{P}^{+}\right)$;

(b) если

$$
\frac{f^{\prime \prime}(q)}{f^{\prime}(q)\left(1-f^{\prime}(q)\right)}=\frac{2}{1-q}
$$

$u f \in \mathscr{E}\left(\mathfrak{P}^{+}\right)$, то $f$ является дробно-линейной функичей.

При $q=0$ результат принимает следующий вид.

СлЕДСТвиЕ 2. Пусть $f(z)=p_{1} z+p_{2} z^{2}+\cdots$ - вероятностная производящая функиия и $p_{1} \neq 1$. Тогда имеют место следующие утверждения:

(a) если точка $\left(p_{1}, p_{2}\right)$ не принадлежит множеству

$$
\left\{(x, y) \in \mathbb{R}^{2}: 0 \leqslant y \leqslant x(1-x), 0 \leqslant x \leqslant 1\right\},
$$

mo $f \notin \mathscr{E}\left(\mathfrak{P}^{+}\right)$;

(b) если $p_{2}=p_{1}\left(1-p_{1}\right)$ u $f \in \mathscr{E}\left(\mathfrak{P}^{+}\right)$, то $f$ является дробно-линейной функиией.

В случае $f(0) \neq 0$ также можно получить необходимые условия вложимости, которые формулируются в терминах начальных вероятностей [81].

Теорема 29. Пусть $f(z)=\sum_{k=0}^{\infty} p_{k} z^{k} \not \equiv z$ принадлежит полугруппе $\mathfrak{P}^{+}[q]$, $0<q \leqslant 1$. Тогда имеют место следующие утверждения:

(a) если точка $\left(p_{0}, p_{1}\right)$ не принадлежит множеству

$$
\left\{(x, y) \in \mathbb{R}^{2}: \frac{1}{q}(q-x)(1-x) \leqslant y<\frac{1}{q}(q-x), 0 \leqslant x<q\right\},
$$

mo $f \notin \mathscr{E}\left(\mathfrak{P}^{+}\right)$; 
(b) если $p_{1}=\left(q-p_{0}\right)\left(1-p_{0}\right) / q u f \in \mathscr{E}\left(\mathfrak{P}^{+}\right)$, то $f$ является дробно-линейной функиией.

Условия вложимости вероятностной производящей функции в однопараметрическую полугруппу в терминах функции Кёнигса были получены в [82].

6.2. Инфинитезимальные преобразования и безграничная делимость в полугруппе вероятностных производящих функций. При описании конуса инфинитезимальных преобразований полугруппы $\mathfrak{P}^{+}$приходится преодолевать две трудности. Во-первых, подполугруппа $\mathfrak{P}^{+}$не является замкнутым в топологии пространства $\mathscr{H}(\mathbb{D})$ подмножеством полугруппы $\mathfrak{P}$. Во-вторых, при воспроизведении однопараметрической полугруппы по инфинитезимальной образующей нужно следить за неотрицательностью коэффициентов тейлоровского разложения функций из однопараметрической полугруппы.

На пути преодоления первой трудности рассмотрим замыкание $\overline{\mathfrak{P}^{+}}$полугруппы $\mathfrak{P}^{+}$в топологии локально равномерной в $\mathbb{D}$ сходимости. Очевидно, что голоморфная в $\mathbb{D}$ функция $f$ принадлежит $\overline{\mathfrak{P}^{+}}$в том и только том случае, если в ее тейлоровском разложении $f(z)=\sum_{k=0}^{\infty} a_{k} z^{k}$ коэффициенты $a_{k}$ удовлетворяют условиям $a_{k} \geqslant 0$ и $\sum_{k=0}^{\infty} a_{k} \leqslant 1$. Таким образом, естественно ставить вопрос об инфинитезимальных преобразованиях полугруппы $\overline{\mathfrak{P}^{+}}$.

Заметим вначале, что для любой функции $f(z)=\sum_{k=0}^{\infty} a_{k} z^{k}$ из $\overline{\mathfrak{P}^{+}}$выполняется неравенство

$$
|f(z)-z| \leqslant 2\left(1-f^{\prime}(0)\right), \quad z \in \mathbb{D} \text {. }
$$

Действительно,

$$
\begin{aligned}
|f(z)-z| & =\left|\sum_{k=0}^{\infty} a_{k} z^{k}-z\right|=\left|\sum_{k=2}^{\infty} a_{k}\left(z^{k}-z\right)+a_{0}(1-z)+(f(1)-1) z\right| \\
& \leqslant(1-f(1))+2 a_{0}+2 \sum_{k=2}^{\infty} a_{k}=\left(1-a_{1}\right)+\left(f(1)-a_{1}\right) \leqslant 2\left(1-a_{1}\right),
\end{aligned}
$$

что эквивалентно (15).

ТеОрема 30. Для того чтобъ голоморфная в $\mathbb{D}$ функиия $v$ представляла собой инфинитезимальную образуюшую однопараметрической полугруппы $t \mapsto f^{t}$ в $\overline{\mathfrak{P}^{+}}$, необходимо и достаточно, чтобы она допускала представление в виде

$$
v(z)=\alpha(h(z)-z)
$$

где $\alpha \geqslant 0$, а функиия $h$ голоморфна в $\mathbb{D}$ и имеет разложение $h(z)=b_{0}+\sum_{k=2}^{\infty} b_{k} z^{k}$, в котором $b_{0}, b_{k} \geqslant 0 u b_{0}+\sum_{k=2}^{\infty} b_{k} \leqslant 1$. 
ДокАЗАтЕльство. Пусть $t \mapsto f^{t}$ - однопараметрическая полугруппа в $\overline{\mathfrak{P}^{+}}$ и $f^{t}(z)=\sum_{k=0}^{\infty} a_{k}(t) z^{k}$. Выясним вид ее инфинитезимальной образующей

$$
v(z)=\lim _{t \rightarrow 0} \frac{f^{t}(z)-z}{t}=\sum_{k=0}^{\infty} c_{k} z^{k} .
$$

Поскольку семейство функций $f^{t}(z), t \geqslant 0$, бесконечно дифференцируемо по $t$ и $f^{t}(z) \rightarrow z$ локально равномерно в $\mathbb{D}$ при $t \rightarrow 0$, то $a_{k}(t), k=0,1,2, \ldots$, также бесконечно дифференцируемы по $t$ и $a_{k}(t) \rightarrow 0$ при $t \rightarrow 0$ и $k \neq 1, a_{1}(t) \rightarrow 1$ при $t \rightarrow 0$. Следовательно, $c_{1} \leqslant 0$ и $c_{k} \geqslant 0$ при $k \neq 1$. Кроме того, переходя в неравенстве

$$
\frac{1}{t}\left(\sum_{k=0}^{n} a_{k}(t)-1\right) \leqslant 0
$$

к пределу при $t \rightarrow 0$, получаем $c_{0}+\sum_{k=2}^{n} c_{k} \leqslant-c_{1}, n=3,4, \ldots$ Отсюда следует, что равенство $c_{1}=0$ влечет $v(z) \equiv 0$ и $f^{t}(z) \equiv z$ при всех $t>0$. Поэтому рассмотрим случай $c_{1}<0$. Тогда $c_{0}+\sum_{k=2}^{\infty} c_{k} \leqslant-c_{1}$ и инфинитезимальную образующую $v$ можно представить в виде (16) с $\alpha=-c_{1}$ и

$$
h(z)=-\frac{c_{0}}{c_{1}}-\sum_{k=2}^{\infty} \frac{c_{k}}{c_{1}} z^{k} .
$$

Необходимость представления инфинитезимальной образующей в виде (16) доказана.

Докажем теперь достаточность представления (16). Не нарушая общности, можно полагать при этом $\alpha=1$, поскольку если $v(z)$ - инфинитезимальная образующая однопараметрической полугруппы $t \mapsto f^{t}$, то $\alpha v(z)$ - инфинитезимальная образующая однопараметрической полугруппы $t \mapsto f^{\alpha t}$.

Итак, пусть $h(z)=b_{0}+\sum_{k=2}^{\infty} b_{k} z^{k}$, где $b_{0}, b_{k} \geqslant 0, b_{0}+\sum_{k=2}^{\infty} b_{k} \leqslant 1$, и $v(z)=h(z)-z$. Для $\lambda \in[0,1]$ введем в рассмотрение функцию

$$
\varphi_{\lambda}(z)=z+\lambda v(z)=\lambda h(z)+(1-\lambda) z .
$$

Очевидно, что $\varphi_{\lambda} \in \overline{\mathfrak{P}^{+}}$. Рассматривая $\mathbb{R}^{+}$как аддитивную полугруппу, выделим в ней подполугруппы $I_{1} \subset I_{2} \subset \cdots$, где $I_{n}$ состоит из чисел вида $t=k \cdot 2^{-n}$, $k=0,1,2, \ldots$, и положим $I=\bigcup_{n=1}^{\infty} I_{n}$. Для каждого $n=1,2, \ldots$ определим функцию $F_{n}: \mathbb{D} \times I_{n} \rightarrow \mathbb{C}$ посредством равенств

$$
F_{n}\left(z, k \cdot 2^{-n}\right)=\varphi_{2^{-n}} \circ \cdots \circ \varphi_{2^{-n}}(z)=\varphi_{2^{-n}}^{k}(z),
$$

$k=0,1,2, \ldots$. Другими словами, $F_{n}\left(\cdot, k \cdot 2^{-n}\right)$ является $k$-кратной итерацией функции $\varphi_{2-n}$. Отображение $t \mapsto F_{n}(\cdot, t)$ является гомоморфизмом, действующим из $I_{n}$ в $\overline{\mathfrak{P}^{+}}$, т. е.

$$
F_{n}(z, s+t)=F_{n}(\cdot, s) \circ F_{n}(z, t)
$$


для всех $s, t \in I_{n}$. Заметим также, что для любого $t \in I$ все функции $F_{n}(\cdot, t)$ определены начиная с некоторого номера. Используя диагональный метод Кантора, выделим подпоследовательность $\left\{F_{n_{j}}\right\}$ такую, что $F_{n_{j}}(z, t) \rightarrow F(z, t)$ локально равномерно в $\mathbb{D}$ при $j \rightarrow \infty$ и всех $t \in I$. Если зафиксировать $t>0$ из $I$, то $t \in I_{n}$ начиная с некоторого номера. При этом в представлении $t=k \cdot 2^{-n}$ число $k$ стремится к бесконечности при $n \rightarrow \infty$. Поэтому из неравенства

$$
F_{n}^{\prime}(0, t)=\varphi_{2^{-n}}^{\prime}\left(\varphi_{2^{-n}}^{k-1}(0)\right) \cdot \varphi_{2^{-n}}^{\prime}\left(\varphi_{2^{-n}}^{k-2}(0)\right) \cdot \varphi_{2^{-n}}^{\prime}(0) \geqslant\left(\varphi_{2^{-n}}^{\prime}(0)\right)^{k}=\left(1-\frac{t}{k}\right)^{k}
$$

следует, что $F^{\prime}(0, t) \geqslant e^{-t}$ для всех $t \in I$. Здесь $F^{\prime}=\partial F / \partial z$. Переходя в равенстве $(17)$ к пределу по подпоследовательности $n_{j}$, приходим к соотношению

$$
F(z, s+t)=F(\cdot, s) \circ F(z, t)
$$

для всех $s, t \in I$. Кроме того, используя неравенство (15), для всех $s<t$ из $I$ получаем

$$
\begin{aligned}
|F(z, t)-F(z, s)| & =|F(\cdot, t-s) \circ F(z, s)-F(z, s)| \\
& \leqslant 2\left(1-F^{\prime}(0, t-s)\right) \leqslant 2\left(1-e^{s-t}\right) .
\end{aligned}
$$

Отсюда следует равномерная относительно $z \in \mathbb{D}$ непрерывность функции $t \mapsto F(z, t)$ на $I$. Это позволяет продолжить $F$ на $\mathbb{D} \times \mathbb{R}^{+}$по непрерывности. Очевидно, что $F(\cdot, t) \in \overline{\mathfrak{P}^{+}}$для всех $t \in \mathbb{R}^{+}$. Из непрерывности операции композиции следует также, что равенство (18) будет выполняться для всех $s, t \in \mathbb{R}^{+}$. Таким образом, $t \mapsto F(\cdot, t)$ - однопараметрическая полугруппа в $\overline{\mathfrak{P}^{+}}$. Выясним, что собой представляет ее инфинитезимальная образующая.

Для удобства определим функции $F_{n}$ на $\mathbb{D} \times \mathbb{R}^{+}$, положив $F_{n}(z, t)=F_{n}(z, k \times$ $\left.2^{-n}\right)$ при $k \cdot 2^{-n} \leqslant t<(k+1) \cdot 2^{-n}, k=0,1,2, \ldots$. Тогда для $t \in I_{n}$ можно записать равенство

$$
F_{n}(z, t)-z=\int_{0}^{t} v \circ F_{n}(z, s) d s .
$$

Действительно, если $t=k \cdot 2^{-n}$, то

$$
\begin{aligned}
F_{n}(z, t)-z & =F_{n}\left(z, k \cdot 2^{-n}\right)-z=\sum_{j=1}^{k}\left(F_{n}\left(z, j \cdot 2^{-n}\right)-F_{n}\left(z,(j-1) \cdot 2^{-n}\right)\right) \\
& =\sum_{j=1}^{k}\left(\varphi_{2^{-n}} \circ F_{n}\left(z,(j-1) \cdot 2^{-n}\right)-F_{n}\left(z,(j-1) \cdot 2^{-n}\right)\right) \\
& =2^{-n} \sum_{j=1}^{k} v \circ F_{n}\left(z,(j-1) \cdot 2^{-n}\right)=\int_{0}^{t} v \circ F_{n}(z, s) d s .
\end{aligned}
$$

Осуществляя в равенстве (19) предельный переход по подпоследовательности $\left\{n_{j}\right\}$, приходим к соотношению

$$
F(z, t)-z=\int_{0}^{t} v \circ F(z, s) d s
$$

для всех $t \in I$. По непрерывности это соотношение распространяется на все $t \in \mathbb{R}^{+}$. Используя его, получаем

$$
\lim _{t \rightarrow 0} \frac{F(z, t)-z}{t}=\lim _{t \rightarrow 0} \frac{1}{t} \int_{0}^{t} v \circ F(z, s) d s=v(z) .
$$


Следовательно, $v(z)=h(z)-z$ является инфинитезимальной образующей однопараметрической полугруппы $t \mapsto F(\cdot, t)$ и теорема доказана.

Общее описание конуса инфинитезимальных преобразований полугруппы $\overline{\mathfrak{P}^{+}}$ можно детализировать в зависимости от точки Данжуа-Вольфа $q$. Заметим вначале, что для замыканий $\overline{\mathfrak{P}_{\mathrm{c}}^{+}}$и $\overline{\mathfrak{P}_{\text {sub }}^{+}}$полугрупп $\mathfrak{P}_{\mathrm{c}}^{+}$и $\mathfrak{P}_{\mathrm{sub}}^{+}$соответственно из теоремы 24 следуют соотношения $\overline{\mathfrak{P}_{\mathrm{c}}^{+}}=\overline{\mathfrak{P}_{\text {sub }}^{+}}=\mathfrak{P}^{+}[1]$. Из теорем 24 и 25 следует также, что $\overline{\mathfrak{P}_{\text {sup }}^{+}}=\overline{\mathfrak{P}^{+}}$. Кроме того, голоморфная в $\mathbb{D}$ функция $f$ принадлежит $\overline{\mathfrak{P}^{+}[q]}, 0 \leqslant q<1$, в том и только том случае, если она допускает представление из теоремы 25, в котором $\lambda_{k} \geqslant 0$ и $\sum_{k=1}^{\infty} \lambda_{k} \leqslant 1$. Таким образом, имеют место следующие результаты (см. [80]).

Теорема 31. Для того чтобъ голоморфная в $\mathbb{D}$ функиия $v$ представляла собой инфинитезимальную образующую однопараметрической полугруппы $t \mapsto f^{t}$ в $\overline{\mathfrak{P}^{+}[q]}, 0 \leqslant q<1$, необходимо и достаточно, чтобъ она допускала представление в виде

$$
v(z)=\alpha\left[q-z+(1-q) \sum_{k=2}^{\infty} \lambda_{k} \frac{z^{k}-q^{k}}{1-q^{k}}\right]
$$

где $\alpha \geqslant 0, \lambda_{k} \geqslant 0 u \sum_{k=2}^{\infty} \lambda_{k} \leqslant 1$.

Заметим также, что если $t \mapsto f^{t}$ - однопараметрическая полугруппа в $\mathfrak{P}^{+}[q]$, то в представлении инфинитезимальной образующей $\sum_{k=2}^{\infty} \lambda_{k}=1$. Случай $q=1$ описывается следующей теоремой.

Теорема 32. Для того чтобы голоморфная в $\mathbb{D}$ функиия $v$ представляла собой инфинитезимальную образующую однопараметрической полугруппы $t \mapsto f^{t}$ в $\mathfrak{P}_{\mathrm{c}}^{+}$(соответственно в $\mathfrak{P}_{\mathrm{sub}}^{+}$), необходимо и достаточно, чтобы она допускала представление в виде

$$
v(z)=\alpha\left[1-z+\sum_{k=2}^{\infty} \frac{\lambda_{k}}{k}\left(z^{k}-1\right)\right],
$$

где $\alpha \geqslant 0, \lambda_{k} \geqslant 0$ u $\sum_{k=2}^{\infty} \lambda_{k}=1$ (соответственно $\left.\sum_{k=2}^{\infty} \lambda_{k}<1\right)$.

Наряду с классом вложимых вероятностных производящих функций представляется естественным ввести также следующее определение.

ОПРЕДЕЛЕНИЕ 4. Будем говорить, что вероятностная производящая функция $f$ является безгранично делимой в полугруппе $\mathfrak{P}^{+}$, если для каждого $n=2,3, \ldots$ найдется такая $f_{n}$ из $\mathfrak{P}^{+}$, что

$$
f(z)=f_{n} \circ \cdots \circ f_{n}(z)=f_{n}^{n}(z),
$$

т. е. $f$ является $n$-кратной итерацией функции $f_{n}$. 
Если $\xi$ - целочисленная случайная величина с вероятностной производящей функцией $f$, то условие безграничной делимости $f$ в полугруппе $\mathfrak{P}^{+}$можно сформулировать как условие на $\xi$ в терминах сумм независимых случайных величин [81]. Это определение введено по аналогии с классическим понятием безгранично делимых распределений вероятности. Важным моментом в описании безгранично делимых распределений оказалось то, что они вложимы в однопараметрические полугруппы мер относительно свертки. Другими словами, в полугруппе вероятностных мер на $\mathbb{R}$ с операцией свертки понятия вложимости и безграничной делимости совпадают.

Теорема 33. Пусть вероятностная производящая функиия $f(z) \not \equiv 1$ является безгранично делимой в полугруппе $\mathfrak{P}^{+}$. Тогда $f$ допускает вложение в однопараметрическую полугруппу в $\mathfrak{P}^{+}$, m. e. $f \in \mathscr{E}\left(\mathfrak{P}^{+}\right)$.

ДокАзАТЕЛЬСтво. Пусть вероятностная производящая функция $f(z)=$ $\sum_{k=0}^{\infty} p_{k} z^{k}$ является безгранично делимой в полугруппе $\mathfrak{P}^{+}$. Покажем, что найдется такая однопараметрическая полугруппа $t \mapsto g^{t}$ в $\mathfrak{P}^{+}$, что $f=g^{1}$. Выделим в доказательстве два случая.

1) Допустим вначале, что $f \in \mathfrak{P}^{+}[q]$ и $0 \leqslant q<1$. Случай $f(z) \equiv z$ тривиален, и поэтому будем в дальнейшем считать, что $f(z) \not \equiv z$. Далее, поскольку $z=q$ является неподвижной точкой для функции $f$ и $p_{k} \geqslant 0$, то $0 \leqslant f^{\prime}(q) \leqslant 1$. Равенство $f^{\prime}(q)=1$ не может достигаться, поскольку это влекло бы тождество $f(z) \equiv z$. Покажем, что и равенство $f^{\prime}(q)=0$ не достигается. Действительно, в случае $q>0$ равенство $f^{\prime}(q)=\sum_{k=1}^{\infty} k p_{k} q^{k}=0$ влечет тождество $f^{\prime}(z) \equiv 0$, из которого следует $f(z) \equiv 1$, что противоречит условиям теоремы. Остается рассмотреть случай $q=0$, т.е. $p_{0}=0, p_{1}=f^{\prime}(0)=0$. Но тогда в представлении $(20)$ функция $f_{n}$ будет также удовлетворять условиям $f_{n}(0)=f_{n}^{\prime}(0)=0$ и разложение этой функции в ряд Тейлора будет начинаться, по крайней мере, с $z^{2}$, а ее $n$-й итерации $f_{n}^{n}-$ со степени $z^{2 n}$. Это означало бы, что $p_{0}=p_{1}=\cdots=p_{2 n}=0$. Поскольку представление (20) возможно для любого $n=2,3, \ldots$, то сделанное предположение $f(0)=f^{\prime}(0)=0$ привело бы к тождеству $f(z) \equiv 0$, что невозможно. Таким образом, $0<f^{\prime}(q)<1$.

Для каждого $n=2,3, \ldots$ определим

$$
\alpha_{n}=n\left(1-f_{n}^{\prime}(q)\right), \quad v_{n}(z)=\frac{f_{n}(z)-z}{1-f_{n}^{\prime}(q)},
$$

где $f_{n}$ из представления (20). Поскольку $f_{n}^{\prime}(q)=\left(f^{\prime}(q)\right)^{1 / n}$, то

$$
\lim _{n \rightarrow \infty} \alpha_{n}=-\ln f^{\prime}(q) .
$$

Далее, заметим, что если $\varphi \in \mathfrak{P}^{+}[q]$ и $0<\varphi^{\prime}(q)<1$, то по теореме $25 \varphi$ можно представить в виде

$$
\varphi(z)=q+(1-q) \sum_{k=1}^{\infty} \lambda_{k} \frac{z^{k}-q^{k}}{1-q^{k}}
$$


где $\lambda_{k} \geqslant 0$ и $\sum_{k=1}^{\infty} \lambda_{k}=1$. При этом $\lambda_{1}=\varphi^{\prime}(0) \leqslant \varphi^{\prime}(q)<1$ и

$$
\frac{\varphi(z)-z}{1-\varphi^{\prime}(q)}=\frac{\psi(z)-z}{1-\psi^{\prime}(q)}
$$

где

$$
\psi(z)=q+(1-q) \sum_{k=2}^{\infty} \lambda_{k}^{*} \frac{z^{k}-q^{k}}{1-q^{k}}, \quad \lambda_{k}^{*}=\frac{\lambda_{k}}{1-\lambda_{1}},
$$

т. е. $\psi \in \mathfrak{P}^{+}[q]$ и $\psi^{\prime}(0)=0$. Таким образом, для $n=2,3, \ldots$ получаем

$$
n\left(f_{n}(z)-z\right)=\alpha_{n} v_{n}(z)=\alpha_{n} \frac{h_{n}(z)-z}{1-h_{n}^{\prime}(q)},
$$

где $h_{n} \in \mathfrak{P}^{+}[q]$ и $h_{n}^{\prime}(0)=0$. В силу принципа компактности можно выбрать подпоследовательность $\left\{n_{j}\right\}$ так, чтобы $h_{n_{j}}(z) \rightarrow h(z)$ локально равномерно в $\mathbb{D}$ при $j \rightarrow \infty$. Очевидно, что предельная функция $h$ принадлежит $\overline{\mathfrak{P}^{+}[q]}$ и $h^{\prime}(0)=0$. Покажем, что $h^{\prime}(q)<1$. Действительно, из представления полугруппы $\overline{\mathfrak{P}^{+}[q]}$ следует, что

$$
h(z)=q+(1-q) \sum_{k=2}^{\infty} \lambda_{k} \frac{z^{k}-q^{k}}{1-q^{k}}
$$

где $\lambda_{k} \geqslant 0$ и $\sum_{k=2}^{\infty} \lambda_{k} \leqslant 1$. Но тогда

$$
h^{\prime}(q)=(1-q) \sum_{k=2}^{\infty} \lambda_{k} \frac{k q^{k-1}}{1-q^{k}} \leqslant(1-q) \frac{2 q}{1-q^{2}}=\frac{2 q}{1+q}<1 .
$$

Здесь мы воспользовались тем, что последовательность $\left\{k q^{k-1} /\left(1-q^{k}\right)\right\}$ монотонно убывает. Это следует из неравенств

$$
\frac{k q^{k-1}}{1-q^{k}}-\frac{(k+1) q^{k}}{1-q^{k+1}}=\frac{q^{k-1}\left(k-(k+1) q+q^{k+1}\right)}{\left(1-q^{k}\right)\left(1-q^{k+1}\right)} \geqslant 0,
$$

$k=2,3, \ldots, 0 \leqslant q<1$.

Таким образом,

$$
\lim _{j \rightarrow \infty} n_{j}\left(f_{n_{j}}(z)-z\right)=\alpha(h(z)-z)
$$

где

$$
\alpha=-\frac{\ln f^{\prime}(q)}{1-h^{\prime}(q)}>0 .
$$

Заметим, что $v(z)=\alpha(h(z)-z)$ является инфинитезимальной образующей некоторой однопараметрической полугруппы $t \mapsto g^{t}$ в $\overline{\mathfrak{P}^{+}[q]}$. Покажем, что $f=g^{1}$. Дифференцируя обе части равенства

по $z$, получаем

$$
\frac{\partial}{\partial t} g^{t}(z)=v\left(g^{t}(z)\right)
$$

$$
\frac{\partial}{\partial t} \ln \left(\left(g^{t}\right)^{\prime}(z)\right)=v^{\prime}\left(g^{t}(z)\right)
$$


где под логарифмом понимается ветвь, принимающая вещественное значение при $z=q$. Интегрирование по $t$ с учетом условия $\left.\left(g^{t}\right)^{\prime}(z)\right|_{t=0} \equiv 1$ дает

$$
\ln \left[\left(g^{t}\right)^{\prime}(z)\right]=\int_{0}^{t} v^{\prime}\left(g^{s}(z)\right) d s .
$$

Равенство вещественных частей записывается в виде

$$
\ln \left|\left(g^{t}\right)^{\prime}(z)\right|=\int_{0}^{t} \operatorname{Re}\left\{v^{\prime}\left(g^{s}(z)\right)\right\} d s .
$$

Поскольку $v^{\prime}(q)=\ln f^{\prime}(q)<0$, то найдется такое $r \in(0,1)$, что в неевклидовом круге

$$
Q=\left\{z \in \mathbb{D}:\left|\frac{z-q}{1-q z}\right| \leqslant r\right\}
$$

будет выполняться неравенство $\operatorname{Re} v^{\prime}(z)<0$. В силу принципа гиперболической метрики, $g^{t}(Q) \subseteq Q$ при всех $t \geqslant 0$. Кроме того, из равенства (21) и способа выбора $Q$ следует неравенство $\left|\left(g^{t}\right)^{\prime}(z)\right| \leqslant 1$ для всех $t \geqslant 0$ и $z \in Q$. Но тогда для любых $z^{\prime}, z^{\prime \prime}$ из $Q$ и $t \geqslant 0$ будет выполняться неравенство

$$
\left|g^{t}\left(z^{\prime}\right)-g^{t}\left(z^{\prime \prime}\right)\right|=\left|\int_{\left[z^{\prime}, z^{\prime \prime}\right]}\left(g^{t}\right)^{\prime}(z) d z\right| \leqslant\left|z^{\prime}-z^{\prime \prime}\right| .
$$

Зафиксируем теперь произвольно $z \in Q$ и проведем следующие оценки:

$$
\begin{aligned}
\mid f(z) & -g^{1}(z)|=| f_{n}^{n}(z)-g^{1}(z) \mid \\
\leqslant & \left|f_{n} \circ f_{n}^{n-1}(z)-g^{1 / n} \circ f_{n}^{n-1}(z)\right|+\left|g^{1 / n} \circ f_{n}^{n-1}(z)-g^{1 / n} \circ g^{(n-1) / n}(z)\right| \\
\leqslant & \left|f_{n} \circ f_{n}^{n-1}(z)-g^{1 / n} \circ f_{n}^{n-1}(z)\right|+\left|f_{n}^{n-1}(z)-g^{(n-1) / n}(z)\right| \\
\leqslant & \left|f_{n} \circ f_{n}^{n-1}(z)-g^{1 / n} \circ f_{n}^{n-1}(z)\right|+\left|f_{n} \circ f_{n}^{n-2}(z)-g^{1 / n} \circ f_{n}^{n-2}(z)\right| \\
& \quad+\left|g^{1 / n} \circ f_{n}^{n-2}(z)-g^{1 / n} \circ g^{(n-2) / n}(z)\right| \leqslant \cdots \\
& \leqslant \sum_{k=0}^{n-1}\left|f_{n} \circ f_{n}^{k}(z)-g^{1 / n} \circ f_{n}^{k}(z)\right| .
\end{aligned}
$$

Отсюда выводим

$$
\begin{aligned}
\max _{z \in Q}\left|f(z)-g^{1}(z)\right| & \leqslant n \max _{z \in Q}\left|f_{n}(z)-g^{1 / n}(z)\right| \\
& =\max _{z \in Q}\left|n\left(f_{n}(z)-z\right)-n\left(g^{1 / n}(z)-z\right)\right| \\
& \leqslant \max _{z \in Q}\left|n\left(f_{n}(z)-z\right)-v(z)\right|+\max _{z \in Q}\left|n\left(g^{1 / n}(z)-z\right)-v(z)\right| .
\end{aligned}
$$

Из предыдущего следует, что правая часть последнего неравенства стремится к нулю, если $n$ стремится к бесконечности по подпоследовательности $\left\{n_{j}\right\}$. Следовательно, $f(z)=g^{1}(z)$ при $z \in Q$. В силу теоремы единственности для аналитических функций получаем $f(z) \equiv g^{1}(z)$, т. е. $f \in \mathscr{E}\left(\mathfrak{P}^{+}\right)$.

2) Рассмотрим теперь случай $f \in \mathfrak{P}^{+}[1]$. Как и в предыдущем случае, будем считать, что $f(z) \not \equiv z$. Снова через $f_{n}$ будем обозначать функции из представления (20). Для них, как и для функции $f$, точка $z=1$ будет точкой Данжуа-Вольфа, т. е. $f_{n} \in \mathfrak{P}^{+}[1], n=2,3, \ldots$. Следовательно, $f_{n}(x)>x$ при 
$0 \leqslant x<1$. Отметим также, что $0<f(0)=a<1$, поскольку $f(z) \not \equiv 1$ и $f(z) \not \equiv z$. Кроме того,

$$
a=f_{n}^{n}(0)=f_{n}\left(f_{n}^{n-1}(0)\right)>f_{n}^{n-1}(0)>\cdots>f_{n}(0) .
$$

Для каждого $n=2,3, \ldots$ определим

$$
\alpha_{n}=n\left(1-f_{n}^{\prime}(a)\right), \quad v_{n}(z)=\frac{f_{n}(z)-z}{1-f_{n}^{\prime}(a)} .
$$

Поскольку

$$
f^{\prime}(0)=\left(f_{n}^{n}\right)^{\prime}(0)=f_{n}^{\prime}\left(f_{n}^{n-1}(0)\right) \cdots f_{n}^{\prime}(0) \leqslant\left(f_{n}^{\prime}(a)\right)^{n},
$$

то $\left(f^{\prime}(0)\right)^{1 / n} \leqslant f_{n}^{\prime}(a)$ и

$$
\alpha_{n}=n\left(1-f_{n}^{\prime}(a)\right) \leqslant n\left(1-\left(f^{\prime}(0)\right)^{1 / n}\right) .
$$

Замечая, что $0<f^{\prime}(0)<1$, получаем ограниченность числовой последовательности $\left\{\alpha_{n}\right\}$. Далее, используя теорему 24 и рассуждения, аналогичные проведенным выше, получаем представление

$$
v_{n}(z)=\frac{h_{n}(z)-z}{1-h_{n}^{\prime}(a)},
$$

где $h_{n} \in \mathfrak{P}^{+}[1]$ и $h_{n}^{\prime}(0)=0, n=2,3, \ldots$. Поскольку $\left|h_{n}^{\prime}(z)\right| \leqslant h_{n}^{\prime}(1) \leqslant 1$, то для любых $z^{\prime}, z^{\prime \prime} \in \mathbb{D}$ и $n=2,3, \ldots$ выполняется неравенство

$$
\left|h_{n}\left(z^{\prime}\right)-h_{n}\left(z^{\prime \prime}\right)\right| \leqslant\left|z^{\prime}-z^{\prime \prime}\right| .
$$

Это означает, что последовательность функций $h_{n}, n=2,3, \ldots$, является равностепенно непрерывной в замыкании $\overline{\mathbb{D}}$ единичного круга $\mathbb{D}$. Используя теорему Арцела и ограниченность последовательности $\left\{\alpha_{n}\right\}$, можно выбрать подпоследовательность $\left\{n_{j}\right\}$ так, чтобы $\alpha_{n_{j}} \rightarrow \alpha$ и $h_{n_{j}}(z) \rightarrow h(z)$ равномерно на $\overline{\mathbb{D}}$ при $j \rightarrow \infty$. В силу теоремы Вейерштрасса предельная функция $h$ будет аналитической в единичном круге и будет принадлежать полугруппе $\mathfrak{P}^{+}[1]$. Кроме того, будет также выполняться условие $h^{\prime}(0)=0$. Из представления

$$
h(z)=1+\sum_{k=2}^{\infty} \frac{\lambda_{k}}{k}\left(z^{k}-1\right),
$$

где $\lambda_{k} \geqslant 0$ и $\sum_{k=2}^{\infty} \lambda_{k} \leqslant 1$, видно, что

$$
h^{\prime}(a)=\sum_{k=2}^{\infty} \lambda_{k} a^{k-1} \leqslant a<1 .
$$

Таким образом, имеем равномерный в $\overline{\mathbb{D}}$ предел

$$
\lim _{j \rightarrow \infty} n_{j}\left(f_{n_{j}}(z)-z\right)=\lim _{j \rightarrow \infty} \alpha_{n_{j}} v_{n_{j}}(z)=\frac{\alpha}{1-h^{\prime}(a)}(h(z)-z)=v(z) .
$$

Предельная функция $v$ является инфинитезимальной образующей однопараметрической полугруппы $t \mapsto g^{t}$ в $\mathfrak{P}^{+}[1]$.

Остается показать, что $f=g^{1}$. Поскольку функции $g^{1 / n}$ для $g^{1}$ играют ту же роль, что функции $f_{n}$ для $f$, то применимы рассуждения, проведенные выше, 
из которых следует выполнение условий теоремы Арцела для последовательности функций $n\left(g^{1 / n}(z)-z\right), n=2,3, \ldots$, в замыкании $\overline{\mathbb{D}}$ единичного круга $\mathbb{D}$. Любая сходящаяся равномерно в $\overline{\mathbb{D}}$ подпоследовательность, выбранная из этой последовательности, должна иметь в качестве предела инфинитезимальную образующую

$$
v(z)=\lim _{t \rightarrow 0} \frac{g^{t}(z)-z}{t} .
$$

Аналогично тому, как было сделано в предыдущем случае, получаем

$$
\max _{z \in \overline{\mathbb{D}}}\left|f(z)-g^{1}(z)\right| \leqslant \max _{z \in \overline{\mathbb{D}}}\left|n\left(f_{n}(z)-z\right)-v(z)\right|+\max _{z \in \overline{\mathbb{D}}}\left|n\left(g^{1 / n}(z)-z\right)-v(z)\right| .
$$

Переходя к пределу по соответствующей подпоследовательности, приходим к равенству $f(z) \equiv g^{1}(z)$. Теорема 33 доказана.

Описание инфинитезимальных преобразований полугрупп вероятностных производящих функций оказалось полезным для исследования задач теории однородных марковских ветвящихся процессов с непрерывным временем. В частности, в [83] были получены оценки для функции распределения момента вырождения процесса. В работе [84] дано полное описание предельных распределений для докритического процесса. Понятие эволюционного семейства вероятностных производящих функций позволило получить некоторые аналитические свойства неоднородных по времени марковских ветвящихся процессов [85].

\section{7. Инверсированное преобразование Коши и некоммутативная вероятность}

В этом разделе мы обозначим еще одно направление, которое интенсивно развивается в последнее время и в котором также естественным образом возникают полугруппы аналитических функций.

Понятие независимости играет центральную роль в классической теории вероятностей. По существу, именно это понятие выделяет теорию вероятностей из общей теории меры. Посредством независимости выделяются важнейшие распределения и случайные процессы. Основным аналитическим аппаратом классической теории вероятностей является метод характеристических функций. В его основе лежит преобразование Фурье

$$
h_{\xi}(u)=\int_{\mathbb{R}} e^{i u x} d \mu(x),
$$

где $\xi$ - случайная величина с распределением вероятностей $\mu$. Решающим фактором этого является то, что преобразование Фурье переводит свертку вероятностных мер в произведение характеристических функций. Свертка вероятностных мер возникает при суммировании независимых случайных величин. Если $\mu$ и $\nu$ - вероятностные меры, отвечающие распределениям независимых случайных величин $\xi$ и $\eta$, то сумма $\xi+\eta$ имеет распределение вероятностей $\mu * \nu$. Это обстоятельство используется при доказательстве центральной предельной теоремы. С понятием свертки связаны такие важные конструкции, как безграничная делимость и однопараметрические полугруппы распределений, процессы с независимыми приращениями. В терминах характеристических 
функций решается вопрос описания безгранично делимых законов. Случайная величина $\xi$ называется безгранично делимой, если для любого натурального $n$ найдутся независимые одинаково распределенные случайные величины $\xi_{n, 1}, \xi_{n, 2}, \ldots, \xi_{n, n}$ такие, что

$$
\xi=\xi_{n, 1}+\xi_{n, 2}+\cdots+\xi_{n, n} .
$$

Термин "безграничная делимость" переносится на вероятностное распределение $\mu$. Для каждого натурального $n$ найдется вероятностная мера $\mu_{n}$ такая, что

$$
\mu=\underbrace{\mu_{n} * \mu_{n} * \cdots * \mu_{n}}_{n \text { раз }} .
$$

Если

$$
h(u)=\int_{\mathbb{R}} e^{i u x} d \mu(x), \quad h_{n}(u)=\int_{\mathbb{R}} e^{i u x} d \mu_{n}(x),
$$

то $h(u)=\left(h_{n}(u)\right)^{n}$ и характеристическая функция $h$ отвечает безгранично делимому распределению в том и только том случае, если существует однопараметрическое семейство характеристических функций $\left\{h_{t}\right\}_{t \geqslant 0}$ такое, что

$$
h_{0}(u) \equiv 1, \quad h_{1}(u)=h(u), \quad h_{t+s}(u)=h_{t}(u) h_{s}(u) .
$$

Другими словами, $h$ вкладывается в однопараметрическую мультипликативную полугруппу характеристических функций. Описание таких однопараметрических полугрупп и составляет формулу Леви-Хинчина (см., например, [86]).

Одной из особенностей квантовой и некоммутативной теории вероятностей является то, что в них имеется не одно, а несколько определений независимости [87]. В квантовой вероятности коммутативная алгебра случайных величин заменяется на некоммутативную алгебру $\mathscr{A}$ операторов, действующих в гильбертовом пространстве $\mathscr{H}$. Роль вероятностной меры играет единичный вектор $\xi \in \mathscr{H}$, который называют состоянием. Вероятностное распределение $\mu$ квантовой случайной величины $X \in \mathscr{A}$ определяется через функциональное исчисление спектральной теории:

$$
\langle\xi, f(X) \xi\rangle=\int_{\sigma(X)} f(z) d \mu(z)
$$

где $\sigma(X)$ - спектр оператора $X$. Мера $\mu$ называется также спектральной мерой, ассоциированной с вектором $\xi$, а $\langle\xi, f(X) \xi\rangle$ может интерпретироваться как математическое ожидание $\mathrm{E} f(X)$. Роль вещественных случайных величин играют самосопряженные операторы $X$, а их распределения вероятностей $\mu$ имеют носители на $\mathbb{R}$. Вероятностные меры с некомпактными носителями соответствуют неограниченным операторам. Если $X$ - положительный оператор, то $\sigma(X) \subset \mathbb{R}^{+}=\{x \in \mathbb{R}: x \geqslant 0\}$, а в случае унитарного оператора $X$ имеет место включение $\sigma(X) \subset \mathbb{T}=\{\varkappa \in \mathbb{C}:|\varkappa|=1\}$.

Обозначим через $\mathscr{M}(\mathbb{R})$ (соответственно $\left.\mathscr{M}\left(\mathbb{R}^{+}\right), \mathscr{M}(\mathbb{T})\right)$ класс всех вероятностных мер на $\mathbb{R}$ (соответственно на $\left.\mathbb{R}^{+}, \mathbb{T}\right)$. Через $\mathscr{M}_{0}^{2}(\mathbb{R})$ обозначим класс вероятностных мер $\mu$ на $\mathbb{R}$, имеющих нулевое среднее и конечную дисперсию, т. e.

$$
\int_{\mathbb{R}} x d \mu(x)=0, \quad \int_{\mathbb{R}} x^{2} d \mu(x)<\infty .
$$


Различные понятия независимости в некоммутативной теории вероятностей приводят к новым операциям в классах вероятностных мер, представляющим собой некоммутативные аналоги свертки. Вместо преобразования Фурье в некоммутативной вероятности аналогичную роль играет так называемое инверсированное преобразование Коши. Посредством этого и родственных преобразований некоторые свертки мер преобразуются в композиции аналитических функций, являющихся образами мер при этих преобразованиях. В результате естественным образом возникают полугруппы аналитических функций и задача их инфинитезимального описания. Коротко опишем некоторые из этих полугрупп, не касаясь алгебраической природы соответствующих видов независимости.

Если $\mu$ - вероятностная мера на $\mathbb{R}$, то под ее преобразованием Коши понимается аналитическая в верхней полуплоскости $\mathbb{U}$ функция $G_{\mu}$, определяемая формулой

$$
G_{\mu}(z)=\int_{\mathbb{R}} \frac{d \mu(u)}{z-u}, \quad z \in \mathbb{U} .
$$

Заметим, что $\mu$ однозначно определяется своим преобразованием Коши $G_{\mu}$ посредством формулы обращения

$$
\mu(B)=-\frac{1}{\pi} \lim _{\varepsilon \searrow 0} \int_{B} \operatorname{Im} G_{\mu}(x+i \varepsilon) d x,
$$

где $B \subset \mathbb{R}$ - борелевское множество, для которого $\mu(\partial B)=0$ (см., например, [88]). Более адекватно отражает вероятностные свойства распределения так называемое инверсированное преобразование Коши

$$
F_{\mu}(z)=\frac{1}{G_{\mu}(z)},
$$

которое представляет собой голоморфное отображение верхней полуплоскости $\mathbb{U}$ в себя. Если $\mu=\delta_{0}$ - мера с единичной массой, сосредоточенной в нуле, то $F_{\mu}(z) \equiv z$ - тождественное преобразование. Обозначим через $\mathfrak{F}$ класс голоморфных преобразований верхней полуплоскости $\mathbb{U}$ в себя

$$
\mathfrak{F}=\left\{f=F_{\mu}: \mu \in \mathscr{M}(\mathbb{R})\right\},
$$

которые представляют собой инверсированные преобразования Коши вероятностных мер $\mu$ на $\mathbb{R}$. Подмножество $\mathfrak{F}_{0}^{2} \subset \mathfrak{F}$ состоит из инверсированных преобразований Коши вероятностных мер $\mu \in \mathscr{M}_{0}^{2}(\mathbb{R})$. В работе [89] было дано теоретико-функциональное описание классов $\mathfrak{F}$ и $\mathfrak{F}_{0}^{2}$. В частности, голоморфная в верхней полуплоскости $\mathbb{U}$ функция $f$, принимающая значения из верхней полуплоскости, принадлежит классу $\mathfrak{F}$ в том и только том случае, если $f(z) \rightarrow \infty$ при $z \rightarrow \infty$ и ее угловая производная на бесконечности равна единице. Это означает, что класс $\mathfrak{F}$ замкнут относительно операции композиции, т. е. является полугруппой. Далее, голоморфная функция $f: \mathbb{U} \rightarrow \mathbb{U}$ принадлежит $\mathfrak{F}_{0}^{2}$ в том и только том случае, если найдется конечная положительная мера $\nu$ на $\mathbb{R}$ такая, что

$$
f(z)=z+\int_{\mathbb{R}} \frac{d \nu(u)}{u-z}, \quad z \in \mathbb{U} .
$$

K этому представлению, т. е. к классу $\mathfrak{F}_{0}^{2}$, приводит выделение в классе Неванлинны (или Пика) подмножества функций с гидродинамической нормировкой 
на бесконечности [49]. В работе [49] было также установлено, что класс $\mathfrak{F}_{0}^{2}$ замкнут относительно операции композиции. Таким образом, $\mathfrak{F}_{0}^{2}$ является подполугруппой полугруппы $\mathfrak{F}$.

Одно из понятий независимости в некоммутативной вероятности, которое переводит соответствующую свертку вероятностных распределений в композицию инверсированных преобразований Коши, было введено Мураки [90].

ОПРЕДЕЛЕНИЕ 5. Пусть $\mu$ и $\nu$ - две вероятностные меры на $\mathbb{R}$ с инверсированными преобразованиями Коши $F_{\mu}$ и $F_{\nu}$ соответственно. Тогда под аддитивной монотонной сверткой $\lambda=\mu \triangleright \nu$ мер $\mu$ и $\nu$ понимается единственная вероятностная мера $\lambda$ на $\mathbb{R}$ с инверсированным преобразованием Коши $F_{\lambda}=F_{\mu} \circ F_{\nu}$.

В связи с изучением аналогов процесса Леви и формулы Леви-Хинчина на основе аддитивной монотонной свертки [91] возникает конструкция однопараметрической полугруппы в $\mathscr{M}(\mathbb{R})$ (или $\left.\mathscr{M}_{0}^{2}(\mathbb{R})\right)$ относительно аддитивной монотонной свертки, которая посредством инверсированного преобразования Коши переводится в композиционную однопараметрическую полугруппу в $\mathfrak{F}$ (или $\left.\mathfrak{F}_{0}^{2}\right)$. Описание инфинитезимальных преобразований полугруппы $\mathfrak{F}_{0}^{2}$ (в контексте голоморфных отображений верхней полуплоскости в себя с гидродинамической нормировкой на бесконечности) было получено в [49].

Теорема 34. Для того чтобы голоморфная в $\mathbb{U}$ функиия $v$ представляла собой инфинитезимальную образующую однопараметрической полугруппь $t \mapsto f^{t}$ в $\mathfrak{F}$, необходимо и достаточно, чтобъ она допускала представление в виде

$$
v(z)=\alpha+\int_{\mathbb{R}} \frac{1+u z}{u-z} d \nu(u)
$$

где $\alpha \in \mathbb{R} u \nu$ - конечная, неотрицательная борелевская мера на $\mathbb{R}$.

ТЕОРема 35. Для того чтобы голоморфная в $\mathbb{U}$ функиия $v$ представляла собой инфинитезимальную образующую однопараметрической полугруппь $t \mapsto f^{t}$ в $\mathfrak{F}_{0}^{2}$, необходимо и достаточно, чтобъ она допускала представление в виде

$$
v(z)=\int_{\mathbb{R}} \frac{d \nu(u)}{u-z},
$$

где $\nu$ - конечная, неотрицательная борелевская мера на $\mathbb{R}$.

Если $\mu$ - вероятностная мера на $\mathbb{R}^{+}$, то вместо преобразования Коши можно определить функцию

$$
\psi_{\mu}(z)=\int_{\mathbb{R}^{+}} \frac{u z}{1-u z} d \mu(u),
$$

которая является аналитической в $\mathbb{S}=\mathbb{C} \backslash \mathbb{R}^{+}$функцией и не принимает значения -1 . Функция $\psi_{\mu}$ называется производящей функцией моментов меры $\mu$. В силу отмеченных свойств $\psi_{\mu}$ можно также определить функцию

$$
\eta_{\mu}(z)=\frac{\psi_{\mu}(z)}{1+\psi_{\mu}(z)},
$$

которая голоморфна в $\mathbb{S}$ и $\eta_{\mu}(\mathbb{S}) \subset \mathbb{S}$. Обозначим через $\mathfrak{T}$ класс голоморфных отображений разрезанной плоскости $\mathbb{S}$ в себя

$$
\mathfrak{T}=\left\{f=\eta_{\mu}: \mu \in \mathscr{M}\left(\mathbb{R}^{+}\right)\right\},
$$


являющихся $\eta$-преобразованиями вероятностных мер $\mu$ на $\mathbb{R}^{+}$. Внутреннее описание класса $\mathfrak{T}$ было получено в работе [92]. Голоморфная функция $f: \mathbb{S} \rightarrow \mathbb{S}$ принадлежит классу $\mathfrak{T}$ в том и только том случае, если

$$
(\operatorname{Im} z)(\operatorname{Im} f(z)) \geqslant 0
$$

для $z \in \mathbb{S}$ и $\lim _{x}>f(x)=0$. Из этого описания видно, что $\mathfrak{T}$ является полугруппой относительно операции композиции. Понятие монотонной свертки на классе мер $\mathscr{M}\left(\mathbb{R}^{+}\right)$ввел Берковичи [93]. В терминах преобразования $\eta_{\mu}$ она определяется следующим образом.

ОПредЕЛЕниЕ 6 . Пусть $\mu$ и $\nu$ - две вероятностные меры на $\mathbb{R}^{+}$с преобразованиями $\eta_{\mu}$ и $\eta_{\nu}$ соответственно. Тогда мультипликативная монотонная свертка мер $\mu$ и $\nu$ определяется как единственная вероятностная мера $\lambda=\mu \circlearrowright \nu$ на $\mathbb{R}^{+}$с преобразованием $\eta_{\lambda}=\eta_{\mu} \circ \eta_{\nu}$.

Таким образом, изучение мультипликативной монотонной свертки переносится на полугруппу аналитических функций $\mathfrak{T}$. Простое преобразование результатов из [44] с учетом того, что $z=0$ является граничной неподвижной точкой, приводит к следующей теореме.

Теорема 36. Для того чтобы голоморфная в $\mathbb{S}$ функиия $v$ представляла собой инфинитезимальную образующую однопараметрической полугруппь $t \mapsto f^{t}$ в $\mathfrak{T}$, необходимо и достаточно, чтобы она допускала представление в виде $v(z)=z h(z)$, где $h$ голоморфна в $\mathbb{S}$,

$$
(\operatorname{Im} z)(\operatorname{Im} h(z)) \geqslant 0
$$

для $z \in \mathbb{S}$ и интеграл

$$
\int_{0} \frac{d u}{u h(-u)}
$$

расходится.

Отметим также, что мультипликативная монотонная свертка на классе мер $\mathscr{M}(\mathbb{T})$ изучалась в работе [94].

\section{Список литературы}

[1] E. Schröeder, "Ueber iterirte Funktionen", Math. Ann., 3:2 (1870), 296-322.

[2] G. Koenigs, "Recherches sur les intégrales de certaines équations fonctionelles", Ann. Sci. École Norm. Sup. (3), 1, suppl. (1884), 1-41.

[3] K. Löwner, "Untersuchungen über schlichte konforme Abbildungen des Einheitskreises. I", Math. Ann., 89:1-2 (1923), 103-121.

[4] L. Bieberbach, "Über die Koeffizienten derjenigen Potenzreihen, welche eine schlichte Abbildung des Einheitskreises vermitteln", S. B. Preuss. Akad. Wiss., 138 (1916), 940-955.

[5] L. de Branges, A proof of the Bieberbach conjecture, LOMI preprints, E-5-84, LOMI, Leningrad, 1984, $21 \mathrm{pp}$.

[6] L. de Branges, "A proof of the Bieberbach conjecture", Acta Math., 154:1-2 (1985), 137-152.

[7] C. Loewner, "Some classes of functions defined by difference or differential inequalities", Bull. Amer. Math. Soc., 56 (1950), 308-319. 
[8] C. Loewner, "On some transformation semigroups", J. Rational Mech. Anal., 5 (1956), 791-804.

[9] C. Loewner, "On some transformation semigroups invariant under Euclidean and non-Euclidean isometries", J. Math. Mech., 8 (1959), 393-409.

[10] C. Loewner, "On semigroups in analysis and geometry", Bull. Amer. Math. Soc., 70 (1964), 1-15.

[11] I. N. Baker, "Fractional iteration near a fixpoint of multiplier 1", J. Austral. Math. Soc., 4:2 (1964), 143-148.

[12] S. Karlin, J. McGregor, "Embedding iterates of analytic functions with two fixed points into continuous groups", Trans. Amer. Math. Soc., 132 (1968), 137-145.

[13] E. Jabotinsky, "Analytic iteration", Trans. Amer. Math. Soc., 108 (1963), 457-477.

[14] G. Szekeres, "Regular iteration of real and complex functions", Acta Math., 100:3-4 (1958), 203-258.

[15] M. Kuczma, Functional equations in a single variable, Monografie Matematyczne, 46, Państwowe Wydawnictwo Naukowe, Warszawa, 1968, 383 pp. (errata insert).

[16] M. Kuczma, B. Choczewski, R. Ger, Iterative functional equations, Encyclopedia Math. Appl., 32, Cambridge Univ. Press, Cambridge, 1990, xx+552 pp.

[17] J. Hadamard, "Two works on iteration and related questions", Bull. Amer. Math. Soc., 50 (1944), 67-75.

[18] Р. Неванлинна, Однозначные аналитические функции, ГИТТЛ, М., 1957; пер. с нем.: R. Nevanlinna, Eindeutige analytische Funktionen, Grundlehren Math. Wiss., XLVI, Springer-Verlag, Berlin-Göttingen-Heidelberg, 1953, x+379 pp.

[19] L. V. Ahlfors, Conformal invariants: topics in geometric function theory, McGraw-Hill Series in Higher Math., McGraw-Hill Book Co., New York-Düsseldorf--Johannesburg, 1973, ix $+157 \mathrm{pp}$.

[20] A. Denjoy, "Sur l'itération des fonctions analytiques", C. R. Acad. Sci. Paris Sér. A, 182 (1926), 255-257.

[21] J. Wolff, "Sur l'itération des fonctions holomorphes dans une région, et dont les valeurs appartiennent à cette région", C. R. Acad. Sci. Paris Sér. A, 182 (1926), 42-43, 200-201.

[22] E. Berkson, H. Porta, "Semigroups of analytic functions and composition operators", Michigan Math. J., 25:1 (1978), 101-115.

[23] В.В. Горяйнов, "Дробные итерации аналитических в единичном круге функций с заданными неподвижными точками", Матем. сб., 182:9 (1991), 1281-1299; англ. пер.: V. V. Goryaĭnov, "Fractional iterates of functions analytic in the unit disk with given fixed points", Math. USSR-Sb., 74:1 (1993), 29-46.

[24] В. В. Горяйнов, О. С. Кудрявцева, "Однопараметрические полугруппы аналитических функций, неподвижные точки и функция Кёнигса”, Матем. сб., 202:7 (2011), 43-74; англ. пер.: V. V. Goryaĭnov, O. S. Kudryavtseva, "One-parameter semigroups of analytic functions, fixed points and the Koenigs function", Sb. Math., 202:7 (2011), 971-1000.

[25] F. Bracci, M. D. Contreras, S. Díaz-Madrigal, "Aleksandrov-Clark measures and semigroups of analytic functions in the unit disc", Ann. Acad. Sci. Fenn. Math., 33:1 (2008), 231-240.

[26] A. G. Siskakis, "Semigroups of composition operators on spaces of analytic functions, a review", Studies on composition operators (Laramie, WY, 1996), Contemp. Math., 213, Amer. Math. Soc., Providence, RI, 1998, 229-252.

[27] J. H. Shapiro, Composition operators and classical function theory, Universitext Tracts Math., Springer-Verlag, New York, 1993, xvi+223 pp.

[28] M. Elin, V. Goryainov, S. Reich, D. Shoikhet, "Fractional iteration and functional equations for analytic in the unit disk", Comput. Methods Funct. Theory, 2:2 (2002), 353-366. 
[29] Ж. Валирон, Аналитические функиии, ГИТТЛ, М., 1957; пер. с фр.: G. Valiron, Fonctions analytiques, Presses Universitaires de France, Paris, 1954, 236 pp.

[30] Ch. Pommerenke, "On the iteration of analytic functions in a halfplane, I", J. London Math. Soc. (2), 19:3 (1979), 439-447.

[31] I. N. Baker, Ch. Pommerenke, "On the iteration of analytic functions in a halfplane, II", J. London Math. Soc. (2), 20:2 (1979), 255-258.

[32] M. D. Contreras, S. Díaz-Madrigal, Ch. Pommerenke, "Fixed points and boundary behaviour of the Koenigs function", Ann. Acad. Sci. Fenn. Math., 29:2 (2004), 471-488.

[33] П.П. Куфарев, “Об однопараметрических семействах аналитических функций”, Матем. сб., 13(55):1 (1943), 87-118.

[34] Ch. Pommerenke, "Über die Subordination analytischer Funktionen", J. Reine Angew. Math., 218 (1965), 159-173.

[35] Ch. Pommerenke, Univalent functions, with a chapter on quadratic differentials by G. Jensen, Studia Mathematica/Mathematische Lehrbücher, XXV, Vandenhoek \& Ruprecht, Göttingen, 1975, 376 pp.

[36] П. П. Куфарев, “Об интегралах простейшего дифференциального уравнения с подвижной полярной особенностью правой части", Уч. зап. Томск. ун-та, 1946, № 1, $35-48$.

[37] D. E. Marshall, S. Rohde, "The Loewner differential equation and slit mappings", J. Amer. Math. Soc., 18:4 (2005), 763-778.

[38] J. R. Lind, "A sharp condition for the Loewner equation to generate slits", Ann. Acad. Sci. Fenn. Math., 30:1 (2005), 143-158.

[39] D. Prokhorov, A. Vasil'ev, "Singular and tangent slit solutions to the Löwner equation", Analysis and mathematical physics, Trends Math., Birkhäuser, Basel, 2009, 455-463.

[40] G.F. Lawler, Conformally invariant processes in the plane, Math. Surveys Monogr., 114, Amer. Math. Soc., Providence, RI, 2005, xii+242 pp.

[41] В. В. Горяйнов, Полугруппы конформных отображений и экстремальные вопросы теории однолистных функций, Дисс. ... докт. физ.-матем. наук, Новосибирск, 1986.

[42] В.Я. Гутлянский, "Параметрическое представление однолистных функций", Докл. АН СССР, 194 (1970), 750-753; англ. пер.: V. Ya. Gutlyanskij, "Parametric representation of univalent functions", Soviet Math. Dokl., 11 (1970), 1273-1276.

[43] В.В. Горяйнов, "О сходимости однопараметрических семейств аналитических функций”, Вопросы метрической теории отображений и ее применение, Наукова думка, Киев, 1978, 13-24.

[44] В.В. Горяйнов, "Полугруппы конформных отображений", Матем. сб., 129:4 (1986), 451-472; англ. пер.: V. V. Goryaŭnov, "Semigroups of conformal mappings", Math. USSR-Sb., 57:2 (1987), 463-483.

[45] У. Рудин, Функииональный анализ, Мир, М., 1975, 443 с.; пер. с англ.: W. Rudin, Functional analysis, McGraw-Hill Series in Higher Math., McGraw-Hill Book Co., New York-Düsseldorf-Johannesburg, 1973, xiii+397 pp.

[46] Р. Эдвардс, Функииональный анализ. Теория и приложения, Мир, М., 1969, 1071 с.; пер. с англ.: R. E. Edwards, Functional analysis. Theory and applications, Holt, Rinehart and Winston, New York-Toronto-London, 1965, xiii+781 pp.

[47] В. В. Горяйнов, "К параметрическому представлению однолистных функций", Докл. АН СССР, 245:5 (1979), 1038-1041; англ. пер.: V. V. Goryaŭnov, "On parametric representation of univalent functions", Soviet Math. Dokl., 20 (1979), 378-381.

[48] G. Schober, Univalent functions - selected topics, Lectures Notes in Math., 478, Springer-Verlag, Berlin-New York, 1975, v+200 pp. 
[49] В.В.Горяйнов, И. Ба, "Полугруппа конформных отображений верхней полуплоскости в себя с гидродинамической нормировкой на бесконечности", Укр. матем. журн., 44:10 (1992), 1320-1329; англ. пер.: V. V. Goryaĭnov, I. Ba, "Semigroup of conformal mappings of the upper half-plane into itself with hydrodynamic normalization at infinity", Ukrainian Math. J., 44:10 (1992), 1209-1217.

[50] B. Gustafsson, A. Vasil'ev, Conformal and potential analysis in Hele-Shaw cells, Adv. Math. Fluid Mech., Birkhäuser Verlag, Basel, 2006, x+231 pp.

[51] Yu. E. Hohlov, D. V. Prokhorov, A. Yu. Vasil'ev, "On geometrical properties of free boundaries in the Hele-Shaw flows moving boundary problem", Lobachevskii J. Math., 1 (1998), 3-12 (electronic).

[52] Г. М. Голузин, Геометрическая теория функиий комплексного переменного, 2-е изд., Наука, М., 1966, 628 с.; англ. пер.: G. M. Goluzin, Geometric theory of functions of a complex variable, Trans. Math. Monogr., 26, Amer. Math. Soc., Providence, RI, 1969, 676 pp.

[53] Г. В. Кузьмина, "Методы геометрической теории функций. I", Алгебра и анализ, 9:3 (1997), 41-103; англ. пер.: G. V. Kuz'mina, "Methods of the geometric theory of functions. I", St. Petersburg Math. J., 9:3 (1998), 455-507.

[54] Г. В. Кузьмина, "Методы геометрической теории функций. II", Алгебра и анализ, 9:5 (1997), 1-50; англ. пер.: G. V. Kuz'mina, "Methods of the geometric theory of functions. II", St. Petersburg Math. J., 9:5 (1998), 889-930.

[55] Н. А. Лебедев, Приниип площадей в теории однолистных функиий, Наука, М., 1975,336 с.

[56] И. М. Милин, Однолистные функции и ортонормированные системъ, Наука, М., 1971, 256 с.; англ. пер.: I. M. Milin, Univalent functions and orthonormal systems, Trans. Math. Monogr., 49, Amer. Math. Soc., Providence, RI, 1977, 202 pp.

[57] М. Лаврентьев, "К теории конформных отображений", Тр. Физ.-матем. ин-та им. В. А. Стеклова, т. 5, Изд-во АН СССР, Л., 1934, 159-245.

[58] M. Schiffer, "A method of variation within the family of simple functions", Proc. London Math. Soc. (2), 44:1 (1938), 432-449.

[59] К. И. Бабенко, "К теории экстремальных задач для однолистных функции класca S", Тр. МИАН СССР, 101 (1972), 3-321; англ. пер.: K. I. Babenko, "The theory of extremal problems for univalent functions of class $S$ ", Proc. Steklov Inst. Math., 101 (1972), 1-327.

[60] Дж. Дженкинс, Однолистные функиии и конформные отображения, ИЛ, М., 1962; пер. с англ.: J. Jenkins, Univalent functions and conformal mapping, Ergeb. Math. Grenzgeb. (N. F.), 18, Reihe: Moderne Funktionentheorie, Springer-Verlag, Berlin-Göttingen-Heidelberg, 1958, vi+169 pp.

[61] И. П. Митюк, "Принцип симметризации для многосвязных областей и некоторые его применения", Укр. матем. журн., 17:4 (1992), 46-54.

[62] A. Baernstein, II, "Integral means, univalent functions and circular symmetrization", Acta Math., 133:1 (1974), 139-169.

[63] W.K. Hayman, Multivalent functions, 2nd ed., Cambridge Tracts in Math., 110, Cambridge Univ. Press, Cambridge, 1994, xii+263 pp.

[64] В.Н. Дубинин, Емкости конденсаторов и симметризачия в геометрической теории функиий комплексного переменного, Дальнаука, Владивосток, 2009.

[65] Г. М. Голузин, "О теоремах искажения в теории конформных отображений", $M a-$ тем. сб., 1(43):1 (1936), 127-135.

[66] L. Bieberbach, "Aufstellung und Beweis des Drehungssatzes für schlichte konforme Abbildungen", Math. Z., 4:3-4 (1919), 295-305.

[67] J. Basilewitsch, "Zum Koeffizientenproblem der schlichten Funktionen", Матем. сб., 1(43):2 (1936), 211-228. 
[68] И.А. Александров, Параметрические продолжения в теории однолистных функций, Наука, М., 1976, 343 с.

[69] P. L. Duren, Univalent functions, Grundlehren Math. Wiss., 259, Springer-Verlag, New York, 1983, xiv+382 pp.

[70] В.В.Горяйнов, "К параметрическому методу теории однолистных функций", Maтем. заметки, 27:4 (1980), 559-568; англ. пер.: V.V. Goryaŭnov, "Parametric method in the theory of univalent functions", Math. Notes, 27:4 (1980), 275-279.

[71] Н.А. Лебедев, "Некоторые оценки для функций, регулярных и однолистных в круге", Вестн. Ленингр. ун-та, 10:11 (1955), 3-21.

[72] В. И. Попов, “Область значений одной системы функционалов на классе $S$ ", $T p$. Томск. ун-та, 182:3 (1965), 106-132.

[73] В. В. Горяйнов, "Об экстремалях в оценках функционалов, зависящих от значений однолистной функции и ее производной", Теория отображений и приближение функиий, Наукова думка, Киев, 1983, 38-49.

[74] В. В. Горяйнов, "Общая теорема единственности и геометрия экстремальных конформных отображений в задачах искажения и вращения", Изв. вузов. Матем., 1986, № 10, 40-47; англ. пер.: V. V. Goryaĭnov, "A general uniqueness theorem and the geometry of extremal conformal mappings in problems of distortion and rotation", Soviet Math. (Iz. VUZ), 30:10 (1986), 54-63.

[75] Т. Харрис, Теория ветвящихся случайных прочессов, Мир, М., 1966, 355 с.; пер. с англ.: Т. E. Harris, The theory of branching processes, Grundlehren Math. Wiss., 119, Springer-Verlag, Englewood Cliffs, N.J.; Prentice-Hall, Inc., 1963, xiv+230 pp.

[76] Б.А. Севастьянов, Ветвящиеся процессы, Наука, М., 1971, 436 с.; нем. пер.: В.A. Sewastjanow, Verzweigungsprozesse, Mathematische Lehrbücher und Monographien. II. Abteilung: Mathematische Monographien, 34, Akademie-Verlag, Berlin, 1974, xi+326 pp.

[77] K. B. Athreya, P.E. Ney, Branching processes, Grundlehren Math. Wiss., 196, Springer-Verlag, New York-Heidelberg, 1972, xi+287 pp.

[78] S. Karlin, J. McGregor, "Embeddability of discrete time simple branching processes into continuous time branching processes", Trans. Amer. Math. Soc., 132 (1968), $115-136$.

[79] T.E. Harris, "Some mathematical models for branching processes", Proceedings of the Second Berkeley Symposium on Mathematical Statistics and Probability, 1950, University of California Press, Berkeley and Los Angeles, 1951, 305-328.

[80] В. В. Горяйнов, “Дробное итерирование вероятностных производящих функций и вложение дискретных ветвящихся процессов в непрерывные", Матем. сб., 184:5 (1993), 55-74; англ. пер.: V. V. Goryaĭnov, "Fractional iteration of probability generating functions and imbedding discrete branching processes in continuous processes", Russian Acad. Sci. Sb. Math., 79:1 (1994), 47-61.

[81] V. V. Goryaŭnov, "Semigroups of probability generating functions, and infinitely splittable random variables", Theory Stoch. Process., 1(17):1 (1995), 2-9.

[82] В. В. Горяйнов, "Функция Кёнигса и дробное итерирование вероятностных производящих функций”, Матем. сб., 193:7 (2002), 69-86; англ. пер.: V. V. Goryaĭnov, "Koenigs function and fractional iterates of probability generating functions", $S b$. Math., 193:7 (2002), 1009-1025.

[83] А. В. Шипилева, "Оценки распределения момента вырождения марковского ветвящегося процесса", ТВП, 45:4 (2000), 776-779; англ. пер.: A. V. Shipileva, "Estimates of the distribution of the extinction moment of a Markov branching process", Theory Probab. Appl., 45:4 (2001), 695-698.

[84] В. В. Горяйнов, А. А. Полковников, "О предельных распределениях вероятностей для докритических ветвящихся процессов", ТВП, 41:2 (1996), 417-425; англ. пер.: 
V. V. Goryaŭnov, A. A. Polkovnikov, "On limit probability distributions for subcritical branching processes", Theory Probab. Appl., 41:2 (1997), 352-359.

[85] V. Goryainov, "Some analytic properties of time inhomogeneous Markov branching processes", Z. Angew. Math. Mech., 76, suppl. 3 (1996), 439-440.

[86] А.Н. Ширяев, Вероятность, в 2-х кн., МЦНМО, М., 2004, 924 с.

[87] N. Muraki, "The five independences as natural products", Infin. Dimens. Anal. Quantum Probab. Relat. Top., 6:3 (2003), 337-371.

[88] Н. И. Ахиезер, И. М. Глазман, Теория линейных операторов в гильбертовом пространстве, изд. 2-е, дополн., Наука, М., 1966, 543 с.; англ. пер. 3-го изд.: N. I. Akhiezer, I. M. Glazman, Theory of linear operators in Hilbert space, v. 9, 10, Monogr. Stud. Math., Pitman (Advanced Publishing Program), Boston-London, 1981, xxxii+312, 313-552 pp.

[89] H. Maassen, "Addition of freely independent random variables", J. Funct. Anal., 106:2 (1992), 409-438.

[90] N. Muraki, Monotonic convolution and monotone Lévy-Hinčin formula, Preprint, Iwate, Japan, 2000.

[91] U. Franz, N. Muraki, "Markov structure of monotone Lévy processes", Infinite dimensional harmonic analysis III (University of Tübingen, Germany, 15-20 September 2003), World Sci. Publ., Hackensack, NJ, 2005, 37-57.

[92] S. T. Belinschi, H. Bercovici, "Partially defined semigroups relative to multiplicative free convolution", Int. Math. Res. Not., 2005:2 (2005), 65-101.

[93] H. Bercovici, "Multiplicative monotonic convolution", Illinois J. Math., 49:3 (2005), 929-951.

[94] U. Franz, "Multiplicative monotone convolutions", Quantum probability, Banach Center Publ., 73, Polish Acad. Sci., Warsaw, 2006, 153-166.

B. B. Горяйнов (V. V. Goryainov)

Волжский гуманитарный институт (филиал)

Волгоградского государственного университета

E-mail: goryainov_vv@hotmail.com
Поступила в редакцию 24.09 .2012 